\title{
INNOVATIVE FOSSIL FUEL FIRED VITRIFICATION TECHNOLOGY FOR SOIL REMEDIATION
}

Contract No. DE-AC21-92MC29120

\section{SUMMARY REPORT \\ PHASE 3 - FULL-SCALE DEMONSTRATION AND PHASE 3A/4 - LIMITED DEMONSTRATION}

REPORTING PERIOD: JUNE 1996 - SEPTEMBER 2001

SUBMITTED TO:

U.S. Department of Energy

National Energy Technology Laboratory

P.O. BOX 880

Collins Ferry Road

Morgantown, West Virginia 26507-0880

\author{
PREPARED BY: \\ VORTEC CORPORATION \\ 3770 RIDGE PIKE \\ CollegeVille, PA 19426 \\ TEL: 610-489-2255 \\ FAX: 610-489-3185
}

PRINCIPAL AUTHORS:

J. Hnat, L.M. BARTONe, M. PinedA

VORTEC REPORT NO. BFA-4110-901-001, REV 1

JULY 13, 2001 


\section{DISCLAIMER}

"This report was prepared as an account of work sponsored by an agency of the United States Government. Neither the United States government nor any agency thereof, nor any of their employees, makes any warranty, expressed or implied, or assumes any legal liability or responsibility for the accuracy, completeness, or usefulness of any information, apparatus, product, or process disclosed, or presents that its use would not infringe privately owned rights. Reference herein to any specific commercial product, process, or service by trade name, trademark, manufacturer, or otherwise does not necessarily constitute or imply its endorsement, recommendation, or favoring by the United States Government or agency thereof. The views and opinions of authors expressed herein do not necessarily state or reflect those of the United States Government or any agency thereof." 


\begin{abstract}
This Summary Report summarizes the progress of Phases 3, 3A and 4 of a waste technology Demonstration Project sponsored under a DOE Environmental Management Research and Development Program and administered by the U.S. Department of Energy National Energy Technology Laboratory-Morgantown (DOE-NETL) for an "Innovative Fossil Fuel Fired Vitrification Technology for Soil Remediation." The Summary Reports for Phases 1 and 2 of the Program were previously submitted to DOE. The total scope of Phase 3 was to have included the design, construction and demonstration of Vortec's integrated waste pretreatment and vitrification process for the treatment of low level waste (LLW), TSCA/LLW and mixed low-level waste (MLLW). Due to funding limitations and delays in the project resulting from a law suit filed by an environmental activist and the extended time for DOE to complete an Environmental Assessment for the project, the scope of the project was reduced to completing the design, construction and testing of the front end of the process which consists of the Material Handling and Waste Conditioning (MH/C) Subsystem of the vitrification plant.
\end{abstract}

Activities completed under Phases 3A and 4 addressed completion of the engineering, design and documentation of the Material Handling and Conditioning System such that final procurement of the remaining process assemblies can be completed and construction of a Limited Demonstration Project be initiated in the event DOE elects to proceed with the construction and demonstration testing of the $\mathrm{MH} / \mathrm{C}$ Subsystem. 


\section{TABLE OF CONTENTS}

\section{$\underline{\text { SECTION }}$}

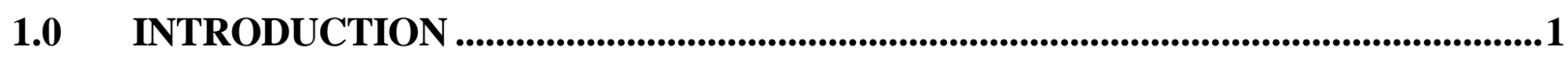

$2.0 \quad$ EXECUTIVE SUMMARY...............................................................................................

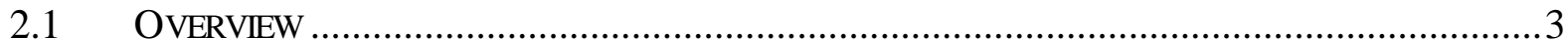

2.2 PROJECT SUMMARY .................................................................................

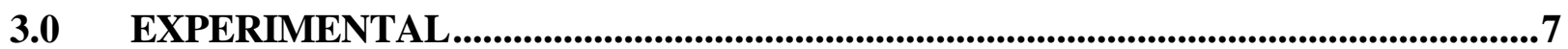

4.0 RESULTS AND DISCUSSION ............................................................................................8

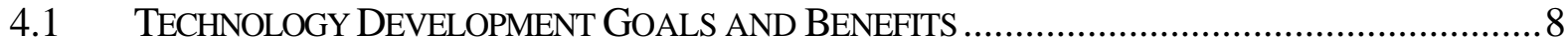

4.1.1 Benefits of Integrated Pretreatment/Vitrification Technology ........................... 8

4.1.2 Benefits of Pretreatment and Conditioning Technology.................................... 9

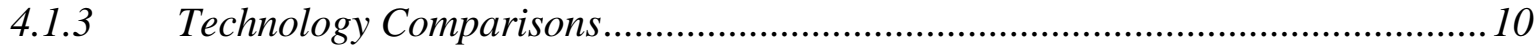

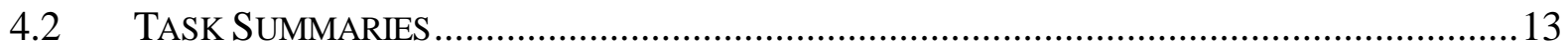

4.2.1 Phase 3 - Integrated Pretreatment/Vitrification System ................................... 13

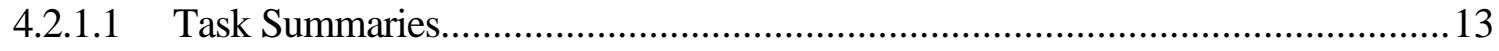

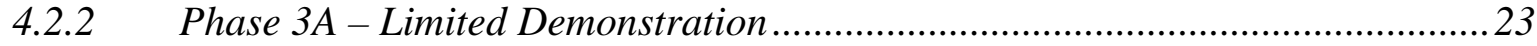

4.2.2.1 Task Summaries........................................................................................ 23

4.2.3 Phase 4-Limited Demonstration.................................................................. 27

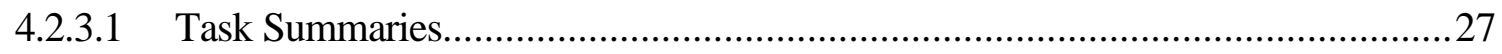

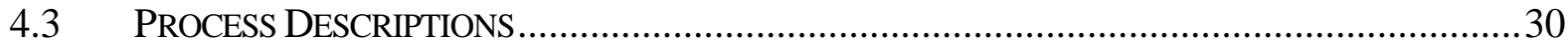

4.3.1 Process Description - Integrated Pretreatment \& Vitrification Plant .................30

4.3.1.1 Feed Preparation Subsystem...................................................................... 33

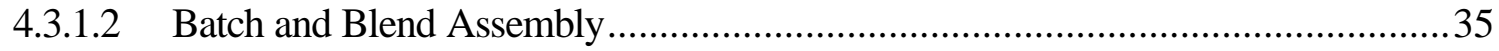

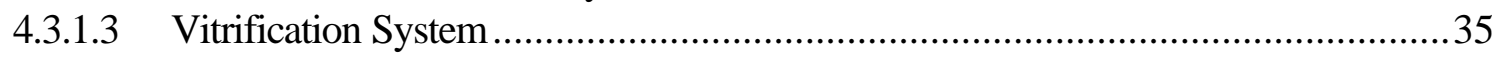

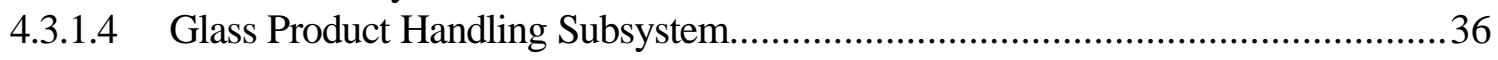

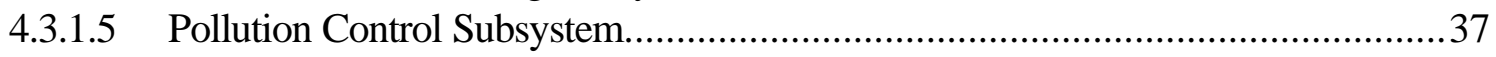

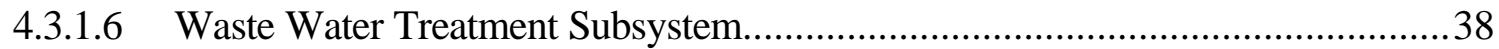

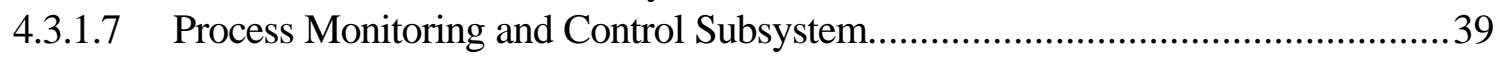

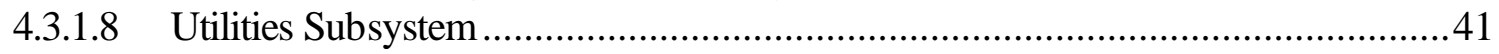

4.3.1.9 Skids/Facility Foundations/Structures/Piping ...............................................42

4.3.2 Limited Demonstration Plant Process Description.............................................44

4.3.2.1 Description of Material Handling and Conditioning System...............................44

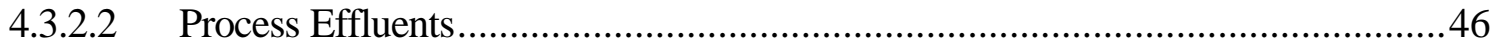

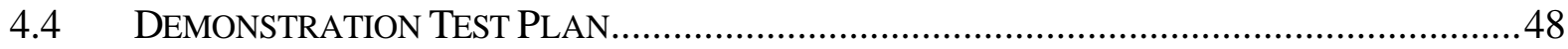

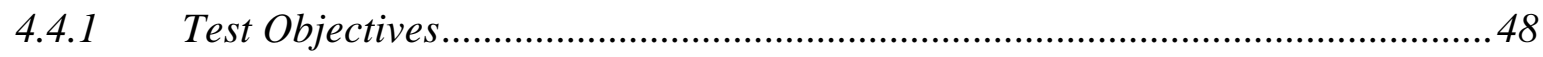

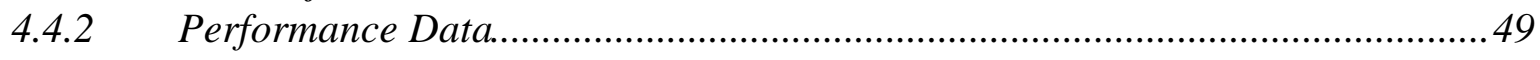

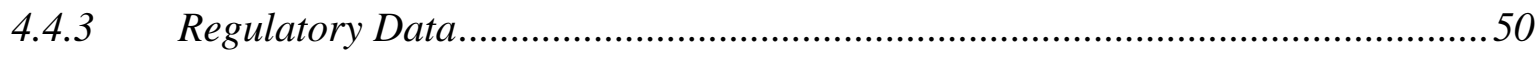

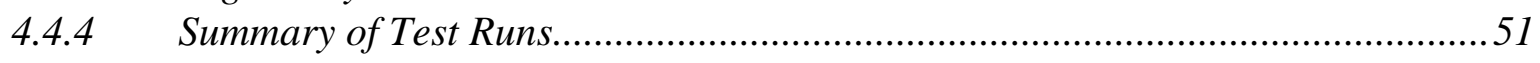




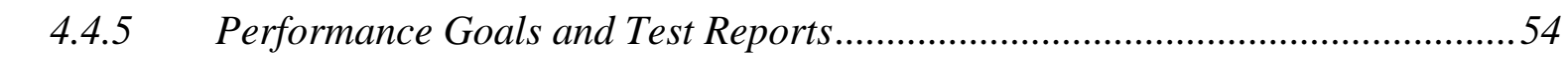

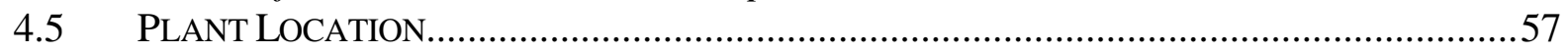

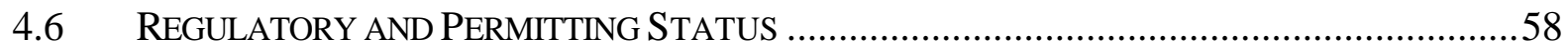

4.6.1 Vitrification Plant - RD\&D and Air Permits...................................................58

4.6.2 Limited Demonstration Plant Permit Requirements..........................................58

5.0 CONCLUSIONS.......................................................................................................................59

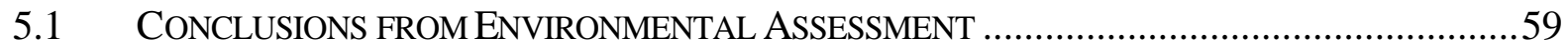

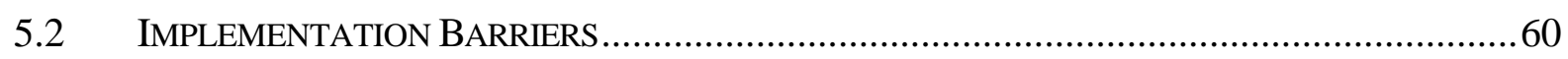

5.3 IMPLEMENTATION OF THE MATERIAL HANDLING AND CONDITIONING SYSTEM ............62

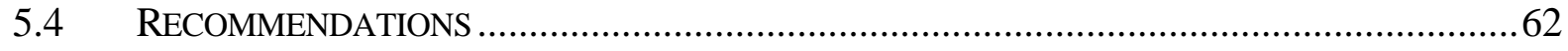

6.0 REFERENCES ..........................................................................................................................63

7.0 BIBLIOGRAPHY ...........................................................................................................64

8.0 LIST OF ACRONYMS AND ABBREVIATIONS...........................................................65

9.0 APPENDICES .......................................................................................................................6 


\section{LIST OF FIGURES}

\section{SECTION}

TITLE

PAGE NO.

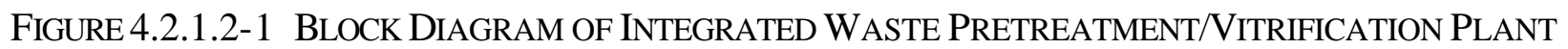

FIGURE 4.2.1.2-2 ARTIST RENDERING OF INTEGRATED WASTE PRETREATMENT/VITRIFICATION PLANT.

FIGURE 4.3.2.1-1. PROCESS FlOW DiAGRAM......

32

FigURE 4.5-1 LOCATION OF DEMONSTRATION FACILITY RELATIVE TO GASEOUS DIFFUSION PLANT

\section{LIST OF TABLES}

SECTION

TITLE

PAGE NO.

TABLE 2.1-1 SuMmary OF GeNERATED, STORED AND BURIED WASTES (METERS ${ }^{3}$ ) AT DOE SiteS

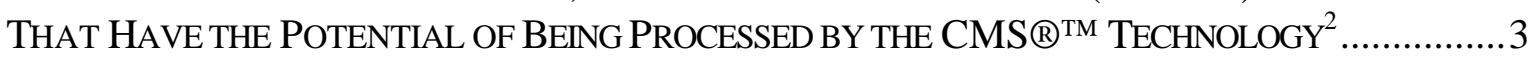

TABLE 4.1.3-1. COMPARISON WITH ALTERNATE TECHNOLOGIES .................................................... 10

TABLE 4.1.3-2. COMPARISON WITH OTHER VITRIFICATION TECHNOLOGIES ................................... 12

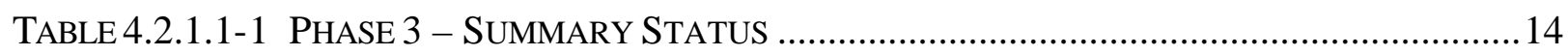

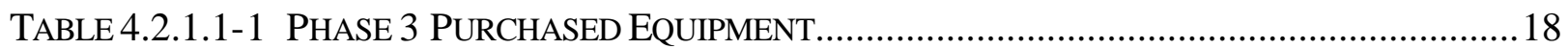

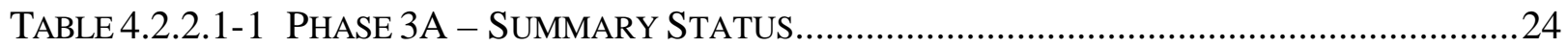

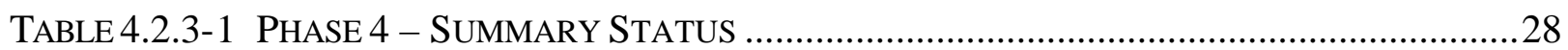

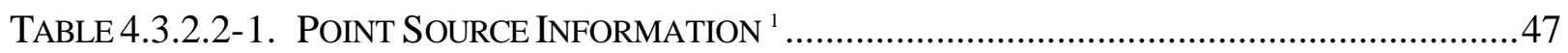

TABLE 4.4.1-1. SUMMARY OF TEST OBJECTIVES.....................................................................49

TABLE 4.4.2-1 PeRFoRMANCE DATA For DEMONSTRATION TRIALS ...........................................50

TABLE 4.4.4-1 TEST OBJECTIVES AND SUPPORTING EXPERIMENTS.............................................51

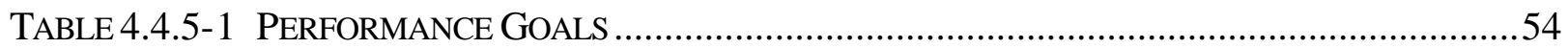

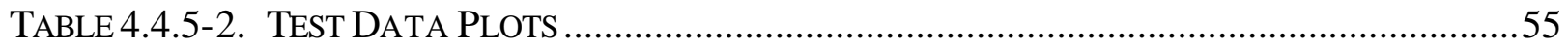

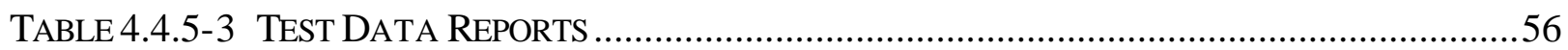

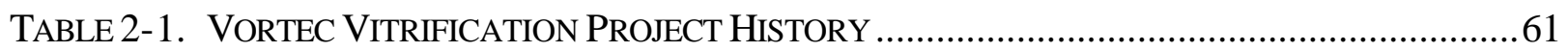




\subsection{INTRODUCTION}

The Department of Energy has been supporting technology developments to allow its goal to clean up its nuclear complex by the year 2019. Achievement of this goal requires the development of innovative technologies that can convert contaminated soils and other wastes containing hazardous and/or radioactive materials into forms that can be safely and readily disposed in accordance with current waste disposal methods.

Drummed wastes can be particularly problematic for treatment and/or disposal because they are typically heterogeneous in nature and the treatment methods must accommodate handling and disposal of the drum containers as well as treatment of the waste contained within the drum. Previous technology development efforts have focused on treatment methods which process waste with minimal conditioning or pre-treatment. However, the operational problems that have evolved as a result of processing heterogeneous wastes have produced some significant system failures and accidents. Significant cost savings in terms of sampling, characterization, handling, transport and disposal can also accrue to waste that are pretreated prior to disposal, even if treatment is not specifically required for disposal.

Vortec Corporation has been developing technologies for DOE applications that both condition waste for efficient waste treatment as well as chemically stabilize the conditioned waste for final, safe, longterm disposal.

This Summary Report summarizes the progress of Phases 3 and 4 of a waste technology Demonstration Project sponsored under a DOE Environmental Management Research and Development Program and administered by the U.S. Department of Energy National Energy Technology Laboratory-Morgantown (DOE-NETL) for an "Innovative Fossil Fuel Fired Vitrification Technology for Soil Remediation." Summary Reports for Phases 1 and 2 of the Program were previously submitted to DOE. The total scope of Phase 3 was to have included the design, construction and demonstration of Vortec's integrated waste pretreatment and vitrification process for the treatment of low level waste (LLW), TSCA/LLW and mixed low-level waste (MLLW). Due to funding limitations and delays in the project resulting from a law suit filed by an environmental activist and the extended time for DOE to complete an Environmental Assessment for the project, the scope of the project was reduced to completing the design, construction and testing of the front end of the process which consists of the Material Handling and Waste Conditioning (MH/C) Subsystem of the vitrification plant.

Activities under Phase 4 focused on completing the design and documentation of the MH/C Subsystem such that final procurement of process assemblies can be completed in the event DOE elects to proceed with the construction and demonstration testing of the MH/C Subsystem. The current tasks for Phase 4 include:

- $\quad$ Task 4.1 - Site Commitment Agreement

The contractor is responsible for establishing and documenting a site commitment agreement for the demonstration and long-term use of the technology. 
- $\quad$ Task 4.2 - Test Plan Development

The contractor shall develop a test plant that will detail the start-up and shakedown testing and continuous operation testing segments of the soil preparation and conditioning subsystems.

- $\quad$ Task 4.3 - Operational Plan

The contractor shall update the design criteria for the soil preparation and conditioning subsystems and document the information in the operational plan in accordance with DOE Order 5480.19.

- $\quad$ Task 4.4 - Final Subsystems Design

The contractor shall complete a detailed final design of the coil preparation and conditioning subsystems based on the preliminary design analysis.

- Task 4.5 - Quality Assurance Plan

The contractor shall be responsible for completing the quality assurance plan for the design, procurement and installation of the soil preparation and conditioning subsystems.

- $\quad$ Task 4.6 - Facility Disposition

The contractor shall be responsible for maintaining the existing equipment from the full-scale demonstration located at the Paducah Gaseous Diffusion plant and at Vortec's pilot plant facility.

Optional tasks under the contract to complete the construction of the demonstration facility and to perform limited demonstration testing may be performed in the future at the option of DOE. The limited demonstration phase of the project is defined as "Phase 4" of the project and is limited to the design, construction and testing of the Material Handling and Conditioning portion of the integrated waste pretreatment and vitrification plant. In designing the $\mathrm{MH} / \mathrm{C}$ System, maximum use was made, to the extent practical, of equipment that had been previously purchased under Phase 3 of the project. The detailed Phase 4 design for the limited demonstration project was developed for specific site conditions at the DOE Gaseous Diffusion Plant located near Paducah, Kentucky. The process, however, is transportable and can, with modifications for site-specific requirements, be used at other DOE facilities. 


\subsection{EXECUTIVE SUMMARY}

\subsection{OVERVIEW}

To accomplish its waste remediation and management missions, the Department of Energy has been evaluating and supporting the development of various technologies. Vitrification and other thermal treatment technologies are being extensively evaluated because of their ability to process a wide variety of organic, heavy metal and radio-nuclide contaminated wastes. The Vortec Cyclone Melting System $(\mathrm{CMS} \circledast)$ vitrification process has the advantage of being very robust with regard to the wastes that can be effectively processed and the spectrum of final glass compositions that can be produced.

Table 2.1-1 summarizes the waste generated annually, stored, and buried at DOE sites that have the potential of being processed using the Vortec CMS®TM technology. These wastes are classified as Low-level, Mixed low-level and TRU waste (TRU must be capable of being contact handle). These waste streams can be processed by the Vortec CMS® at costs lower than the cost of the alternatives being considered by DOE (Report INEL-95/0129, "Integrated Thermal Treatment System Study"). The principal reason for this reduced cost is related to the CMS®'s ability to handle a variety of LLW and MLLW waste streams simultaneously. At Paducah for instance, the LLW is the principle agent for glass forming constituents and combines the processing of LLW and MLLW into the same operation.

Table 2.1-1 Summary of Generated, Stored and Buried Wastes (meters ${ }^{3}$ ) at DOE Sites That Have the Potential of Being Processed by the CMS® Technology ${ }^{2}$

\begin{tabular}{|lccc|}
\hline & Generated Annually & Previously Stored & Previously Buried \\
\hline Low-level & 29,199 & 245,775 & $3,152,456$ \\
Mixed Low-level & 7,959 & 34,259 & $3,011,459$ \\
TRU & 1,288 & 73,537 & 140,997 \\
\hline Total & $\mathbf{3 8 , 4 4 6}$ & $\mathbf{3 5 3 , 5 7 1}$ & $\mathbf{6 , 3 0 4 , 9 1 2}$ \\
\hline
\end{tabular}

Reference 2: DOE/RW-0006, Rev 12, "Integrated Data Base Report-1995”

Significant cost savings can accrue the U.S. Government through the implementation of the pretreatment and vitrification technologies under development. Based on analysis of the waste management tasks currently practiced by DOE (i.e., waste supervision/maintenance, sampling, characterization, handling, treatment, transport and disposal), it is estimated that implementation of these technologies could result in DOE waste management savings in the range of hundreds of millions of dollars. The wastes at Paducah alone include more than 50,000 drums of legacy waste and more than 250,000 cubic meters of remediation wastes which need to be treated and/or disposed.

This report summarizes the progress being made in Phases 3 and 4 of the demonstration of a waste pretreatment and vitrification plant using Vortec's CMS ${ }^{\mathrm{TM}}$ technology at the Paducah Gaseous Diffusion Plant (PGDP). Successful implementation of the waste pretreatment and/or CMS® technologies will significantly increase the rate at which LLW and MLLW waste can be processed. This increased rate will translate into reduced clean-up costs to DOE. The Paducah 
Gaseous Diffusion Plant (PGDP) was selected for this demonstration because of its expressed interest in the technology, the impact the demonstration will have on the remediation effort at the site, and the site's willingness to participate in the financial support of the project. This DOE vitrification demonstration project has progressed through the detailed design and procurement of portions of the pretreatment/vitrification plant.

Phase 1 consisted of pilot scale testing with surrogate wastes and the conceptual design of a process plant for a generic DOE waste stream.

The objective of Phase 2 was to develop a process plant design for the treatment of wastes at a specific DOE facility, namely, PGDP. During Phase 2, a site specific conceptual design was developed for the processing of LLW soils and mud containing TSCA organics and RCRA metal contaminants.

Vitrification trials were conducted during Phase 2 at Vortec's pilot scale vitrification plant located at the University of Pittsburgh Advanced Research Center in Harmarville, PA. The sampling of the effluent and influent streams taken during the tests confirmed that virtually all of the refractory radio-nuclides were retained in the glass and would not leach to the environment - as confirmed by both Product Consistency Tests (PCT) and Toxicity Characteristic Leaching Procedure (TCLP) testing. The organic contaminant was destroyed during testing with a Destruction and Removal Efficiency (DRE) of at least 99.99\%, and semi-volatile RCRA metal surrogates were captured by the Air Pollution Control (APC) system. The data generated during these pilot tests relating to the partitioning of the contaminants throughout the system helped established the Demonstration Plant's design criteria.

Phase 3 included the design of a full scale demonstration at the DOE Paducah Gaseous Diffusion Plant and the procurement of portions of the equipment for this facility. During Phase 3, the basic vitrification process design was modified to meet the specific needs of the waste streams available at Paducah. The system design developed for Paducah significantly enhanced the processing capabilities of the Vortec vitrification process. The overall system design, after several design iterations and component tests at vendor sites, now includes the capability to shred entire drums and drum packs containing mud, concrete, plastics and PCB's and has been modified for processing bulk waste materials. This enhanced processing capability will substantially expand the total DOE waste remediation applications of the technology.

Phase 4 includes the final design and documentation for the procurement installation of major elements of the front end of the process that is designated as the Material Handling and Conditioning System. This phase of the project includes options to complete construction and limited demonstration testing of the Material Handling and Conditioning System. The design and documentation portion of Phase 4 is scheduled to be completed in September 2001. With prompt execution of the options, the construction and demonstration testing can be completed in FY 2002. Because the demonstration is effectively full scale, operational implementation could also occur in late CY 2002 or early 2003.

\subsection{PROJECT SUMMARY}


The original project was awarded in 1992. Phase 1 (feasibility study) and Phase 2 (pilot testing and conceptual design) were successfully completed in 1995. Authorization to proceed with Phase 3 was granted in 1996. During Phase 1, Vortec demonstrated the CMS® technology's ability to remediate a generic soil contaminated with radio-nuclides, heavy metals, and organic materials. Vortec also developed a preliminary design of a CMS ${ }^{\circ}$ based pretreatment/vitrification plant for remediating soil contaminated with heavy metals, radio-nuclides, and organic contaminants. During Phase 2, surrogate soils were defined using data obtained from Hanford's 116-D-1B, 116-F-4, and 300-FF-1 areas, and these soils were vitrified at the Vortec pilot facility. In addition, the preliminary design completed during Phase 1 was expanded and modified into a final design for a CMS $®$ system capable of processing 25 TPD (See Summary Report for Phase 2, January 1, 1995.).

The Paducah's Gaseous Diffusion Plant was selected as the site for the Phase 3 demonstration portion of the project. The principal objective of Phase 3 was to complete engineering, fabricate, install, and operate, for a period of approximately 30 days, a CMS® system designed to vitrify actual soil contaminated with heavy metals and/or low-level radioactivity. The capability to process PCB's and TCE's was also included in the design, but was scheduled for a subsequent phase of the demonstration due to the need for TSCA permit.

During 1996 through 1997, engineering continued to the point where Vortec was ready to proceed into construction. Site preparation work was completed, including excavation of unconsolidated fill materials, placement of geo-textiles, and construction of concrete pads for the equipment. Because it represented a research and development project limited in duration and scope (i.e., less than 2 years and handling approximately $10 \%$ of Paducah's legacy waste), DOE originally determined that the demonstration facility would be eligible for a categorical exclusion as a temporary pilot-scale waste treatment facility under 10 CFR Part 1021, Subpart D. This approach was challenged via a citizen lawsuit alleging that the treatment activities triggered certain requirements under the National Environmental Policy Act (NEPA). Under a consent agreement executed in 1997, DOE agreed to prepare an Environmental Assessment (EA) for the Demonstration Project and further agreed to discontinue site work during this time.

During 1997 through early 2000, much of the planned work was postponed while DOE prepared the EA for the Phase 3 demonstration program in fulfillment of the terms of a 1997 consent agreement. DOE issued the final approved EA and a Finding of No Significant Impact to the environment (FONSI) on March 8, 2000. The issuance of the EA and FONSI fulfilled the requirements of the 1997 consent agreement, which should have allowed DOE and Vortec to complete the Phase 3 Demonstration. However, a second stakeholder lawsuit, challenging the EA and the FONSI, was filed shortly thereafter and this lawsuit caused continuance of the work delays. This lawsuit was settled in late 2000, with the condition (among others) that no thermal treatment system would be installed at Paducah for one year

In a contract modification issued in April 2000, DOE directed Vortec to prepare a cost proposal for a limited demonstration of Vortec's soil preparation and conditioning subsystems, designated Phase 3A to distinguish it from the previous scope of work. The scope included construction, shakedown, startup and demonstration testing, using surrogate materials, of Vortec's soil preparation and conditioning subsystems at the DOE Paducah Gaseous Diffusion Plant (PGDP). In August 2000, Vortec was 
directed to continue with engineering in support of this Limited Demonstration. Contract definitization was delayed pending settlement of the most recent lawsuit. This suit was settled in November 2000.

During the remainder of 2000 and the first two months of 2001, Vortec completed the majority of engineering and much of the procurement work for the Limited Demonstration. Proposal VC-00-10 with estimated cost for the limited demonstration project of approximately $\$ 5.7$ million was submitted to DOE on February 23, 2001. DOE subsequently instructed Vortec to complete the design for the limited demonstration facility and to complete this Summary Report and related design, test and operational procedures under Phase 4 of the project. Phase 4 includes a scope of work change that limits the demonstration to the Material Handling and Conditioning portion of the plant. 


\subsection{EXPERIMENTAL}

No experimental data was developed under Phases 3 and 4 of this project. For the experimental portion of the project, the reader is referred to the Phase 2 Summary Report, dated January 1, 1995. 


\subsection{RESULTS AND DISCUSSION}

\subsection{TECHNOLOGY DEVELOPMENT GOALS AND BENEFITS}

The Department of Energy's goal in pursuing the this technology development program is to develop an innovative technology to convert soils contaminated by hazardous and/or radioactive wastes to forms that can be readily disposed in accordance with current waste disposal methods. The Vortec vitrification technology is able to accomplish this task with minimum public and occupational health risks, with minimum environmental risks, and in a timely and economical manner. Additionally, the vitrification technology transforms the hazardous and/or radioactive waste into a form that has long-term chemical stability to prevent migration of RCRA metals and radio-nuclides, and can thus be disposed in an environmentally safe manner; and which satisfies all federal, state, and local emissions regulations. The organic compounds in the waste are effectively destroyed with a DRE of $99.99 \%$ or greater.

The objective of the demonstration facility is to determine the Vortec technology's performance under full scale field conditions and to fully characterize its operational, technical, financial, and environmental performance for a potential variety of different types of DOE legacy wastes. Originally, testing was to be done with actual waste materials; however, because of permitting and other cost impacted issues, the testing will be done with non-hazardous surrogate materials. The integrated operation of equipment, control systems, and residuals will be evaluated with the specific wastes surrogates that are candidates for future treatment. The details of the testing with surrogate materials is discussed more fully in the project Test Plan which is a separate deliverable.

\subsubsection{Benefits of Integrated Pretreatment/Vitrification Technology}

The unique features of the proposed integrated pretreatment/vitrification technology should make it a particular that contain contaminated soils, sediments, sludges, and mill tailings, organic, metallic, and/or radioactive contaminants.

Benefits for DOE include:

- Immobilization of heavy metals, toxic inorganics, and radionuclides over the long-term.

- Destruction of toxic and/or hazardous organic compounds such as PCBs and TCEs.

- $\quad$ Processing flexibility with regard to the types of solid wastes materials which can be processed in drums or in bulk to accommodate variations in feedstock composition and provision for a broad range of hazardous material remediation applications in a single unit.

- $\quad$ Remediation of materials introduced as in slurry form and the capability for mixing contaminated or waste organic liquids with various types of hazardous solids, such as soils, soil wash process sediments, and mill tailings. The CMS $®$ has demonstrated the capability of processing soilwater slurry and water-soluble waste streams. 
- Minimization of leakage of contaminated gases to the atmosphere via a sealed process, which can be operated at negative pressure.

- Safe remote/automated handling and sampling of the waste..

- $\quad$ Reduction in the decommissioning and disposal costs of the process, due to its small physical size.

\subsubsection{Benefits of Pretreatment and Conditioning Technology}

The pretreatment and conditioning technology has significant benefits by itself in that waste materials can be prepared for further treatment by a variety of thermal or non-thermal processes and/or directly disposed with substantially reduced sampling, characterization, handling, transport and disposal costs.

In modifying the scope to demonstrate the Soil Preparation and Conditioning Facilities, DOE gains several stand-alone benefits, including the utilization of its capital investment in the Phase 3 equipment. The pretreatment facilities are anticipated to yield a substantial cost savings over storing, transporting, and disposing drums by:

(1) Reducing waste sampling costs by eliminating intensive manual operations and using automated sampling techniques.

(2) Reducing characterization costs by the homogenization of discrete waste streams and repackaging the waste into bulk containers, thus providing more accurate characterization with fewer samples.

(3) Lowering transportation costs by efficiently repackaging the waste in bulk containers, thus reducing handling costs and allowing greater volumes to be shipped on transport carriers.

(4) Reducing disposal cost by reduction of the waste volume and the number of containers to be handled.

(5) Reducing disposal cost by removing free liquids from the waste, thus ensuring waste acceptance criteria are satisfied.

(6) Facilitating treatment (if required) by providing a sized, homogeneous waste form for final treatment.

(7) Reduction of long-term drum storage and oversight costs.

Other potential benefits include:

- Reduced risk of exposure to workers and the public.

- Control of fugitive emissions and mitigation of risk to human health and the environment.

- The basic process is designed to handle drummed and containerized waste; however, the system can be readily modified to handle certain bulk wastes as needed.

It is estimated that the implementation of the waste pre-treatment and conditioning technology will save more than $\$ 400$ million across the DOE complex with additional substantial savings for FUSRAP and DOD projects. The system, when constructed, will be available to solve problems where radioactive and/or hazardous waste contained in drums or in certain bulk forms must be removed for treatment or disposal. 


\subsubsection{Technology Comparisons}

The data presented in Table 4.1.3-1 is a qualitative comparison of alternate remediation technologies, for DOE applications. The comparisons presented are for landfill, incineration, stabilization and vitrification alternatives. From the comparisons presented in Table 4.1.3-1, vitrification technology is judged to be superior with regard to its ability to produce a vitrified product (final waste form) and has the highest level of chemical stability with the ability to contain inorganic and radioactive contaminates for tens of thousands of years . In addition, vitrification processes also effectively destroy organic compounds because of the high operating temperatures and residence times at these temperatures.

Table 4.1.3-2 presents a comparison of different classes of vitrification technologies. The data indicates that major advantages of the $\mathrm{CMS} \AA$ technology are its low operating/maintenance cost, its high throughput capacity and its operational robustness while being able to produce a product that meets or exceeds all of the applicable product quality control and leaching criteria. The CMS $®$ has been demonstrated not to be as sensitive to variations in the waste stream's chemical composition as other vitrification processes.

Table 4.1.3-1. Comparison with Alternate Technologies 


\begin{tabular}{|c|c|c|}
\hline Alternatives & Advantages & Disadvantages \\
\hline Landfilling & Low initial capital investment & $\begin{array}{l}\text { No waste volume reduction } \\
\text { Does not destroy organic compounds } \\
\text { Does not stabilize metals \& radionuclides } \\
\text { Requires long term monitoring } \\
\text { Has significant potential for ground water } \\
\text { contamination } \\
\text { Does not diminish generator long term liability } \\
\text { Low potential for resource recovery }\end{array}$ \\
\hline Incineration & \begin{tabular}{|l|} 
Reduces waste volume \\
Destroys organic compounds
\end{tabular} & $\begin{array}{l}\text { Preferred application is high organic content wastes } \\
\text { Generates substantial residuals } \\
\text { Residuals have leaching problems } \\
\text { Requires air pollution control } \\
\text { Hostile regulatory environment } \\
\end{array}$ \\
\hline Vitrification & \begin{tabular}{|l|} 
Reduces waste volume \\
Destroys organic compounds \\
Stabilizes inorganic contaminants \\
Products consistently pass TCLP and PCT \\
Long term product stability \\
Minimal long term generator liability \\
Reduced life cycle cost
\end{tabular} & $\begin{array}{l}\text { Will require landfill of vitrified product only if } \\
\text { radioactive (otherwise a value added product can be } \\
\text { generated) } \\
\text { Requires some waste separation or pretreatment } \\
\text { Requires air pollution control }\end{array}$ \\
\hline Stabilization & $\begin{array}{l}\text { Reduces landfill liability } \\
\text { Low initial capital cost }\end{array}$ & $\begin{array}{l}\text { Significantly increases waste volume } \\
\text { No guarantee of effectiveness } \\
\text { Requires landfill monitoring } \\
\text { No reduced long-term generator liability } \\
\text { No significant life cycle cost advantage } \\
\end{array}$ \\
\hline
\end{tabular}


Table 4.1.3-2. Comparison with Other Vitrification Technologies

\begin{tabular}{|c|c|c|}
\hline \begin{tabular}{|l|} 
Alternatives \\
\end{tabular} & Advantages & Disadvantages \\
\hline \begin{tabular}{|l|} 
Joule \\
Heating
\end{tabular} & $\begin{array}{l}\text { Destroys organics } \\
\text { Stabilizes inorganics }\end{array}$ & $\begin{array}{l}\text { Expensive (cost of electrical power \& maintenance) } \\
\text { Low throughput capacity } \\
\text { Potential volume increase } \\
\text { Effectiveness limited by metals contamination, moisture, } \\
\text { and carbon/organic content } \\
\text { Phase separation is common } \\
\text { Accelerated refractory wear } \\
\text { Requires air pollution control } \\
\text { Requires waste preparation } \\
\text { Organics may require post combustion }\end{array}$ \\
\hline \begin{tabular}{|l|} 
CMS $®$ \\
Technology
\end{tabular} & $\begin{array}{l}\text { Very high throughput capacity } \\
\text { Destroys organics } \\
\text { Stabilizes inorganic contaminants } \\
\text { Organics contribute to energy source } \\
\text { Effective treatment of solids, liquids \&gases } \\
\text { Multi-fuel capability } \\
\text { Low operating and maintenance cost } \\
\text { Produces a homogeneous product without } \\
\text { phase separation } \\
\text { Low operating and maintenance cost }\end{array}$ & $\begin{array}{l}\text { Requires waste preparation } \\
\text { Requires air pollution control }\end{array}$ \\
\hline \begin{tabular}{|l|} 
Molten \\
Metal \\
Processes
\end{tabular} & \begin{tabular}{|l} 
Stabilizes waste \\
Provides volume reduction \\
Can process organics and metals
\end{tabular} & $\begin{array}{l}\text { Very expensive } \\
\text { Very little operational data available } \\
\text { High maintenance and refractory wear } \\
\text { Phase separation inhomogeneous products } \\
\text { Requires air pollution control } \\
\text { Requires waste preparation } \\
\text { Requires post oxidation or after burning } \\
\text { High metals carryover }\end{array}$ \\
\hline $\begin{array}{l}\text { Plasma and } \\
\text { Electric Arc } \\
\text { Processes }\end{array}$ & $\begin{array}{l}\text { Provides volume reduction } \\
\text { Can treat solids, liquids or gases }\end{array}$ & $\begin{array}{l}\text { High operating and maintenance costs } \\
\text { Low throughput capacity } \\
\text { Produces inhomogeneous products } \\
\text { Product leaching problems } \\
\text { Incomplete destruction of organics } \\
\text { Requires air pollution control } \\
\text { Requires post oxidation or after burning } \\
\text { Inefficient energy utilization } \\
\text { Limited applicability }\end{array}$ \\
\hline
\end{tabular}




\subsection{TASK SUMMARIES}

The results and discussions for this subsection are broken down into three sections. Section 4.2.1 addresses the results relating to the design and construction of an integrated waste pretreatment/vitrification plant (Phase 3). Section 4.2.2 summarizes the results and progress relating to the final design, construction and testing a demonstration facility which is limited to the Material Handling and Conditioning portion of the integrated plant (Phase 3A). Section 4.2.3 describes the status of the project relating to the final design, construction and testing of the Material Handling and Conditioning System under Phase 4 of this project.

\subsubsection{Phase 3 - Integrated Pretreatment/Vitrification System}

Phase 3 included five major tasks to be completed. These included:

$\begin{array}{lll}\text { - } & \text { Task 3.1 } & \text { Test System Design Criteria Definition } \\ \text { - } & \text { Task 3.2 } & \text { Component Design and Fabrication } \\ \text { - } & \text { Task 3.3 } & \text { Integrated System Design } \\ \text { - } & \text { Task 3.4 } & \text { Integrated System Facility Construction } \\ \text { - } & \text { Task 3.5 } & \text { Integrated System Testing }\end{array}$

\subsubsection{Task Summaries}

The status of work activities performed under Phase 3 are summarized in Table 4.2.1-1 below. Additional narrative descriptions of the Phase 3 task activities are provided in ensuing sections of this report. The estimated level of completion for Phase 3 work as of the initiation of Phase 3A is also summarized in this table. All work on the task items relating to design and testing of the integrated pretreatment/vitrification plant was suspended in May 2000 because contract modification (A040) redirected work to activities related to Limited Demonstration tasks only. 
Table 4.2.1.1-1 Phase 3 - Summary Status

\begin{tabular}{|c|c|c|}
\hline Task & Task Scope/Deliverable & Status/Remarks \\
\hline \multirow[t]{4}{*}{3.1 Test System Design Criteria } & Update of Criteria from Phase 2 & Completed \\
\hline & Operational Plan & $50 \%$ Complete \\
\hline & $\begin{array}{l}\text { Operational Health and Safety } \\
\text { Plan }\end{array}$ & Completed \\
\hline & Quality Assurance Plan & Completed \\
\hline \multirow[t]{2}{*}{$\begin{array}{l}\text { 3.2 Full Scale Component } \\
\text { Design and Fabrication }\end{array}$} & $\begin{array}{l}\text { Equipment \& Material } \\
\text { Specifications \& Drawings }\end{array}$ & $75 \%$ Complete \\
\hline & Equipment Procurement & 75\% Complete \\
\hline $\begin{array}{l}\text { 3.3 Full Scale Integrated System } \\
\text { Design }\end{array}$ & Final Design \& Documentation & $75 \%$ Complete \\
\hline \multirow{2}{*}{$\begin{array}{l}\text { 3.4 Full Scale Integrated } \\
\text { Construction }\end{array}$} & Site Preparation Activities & 90\% Completed \\
\hline & $\begin{array}{l}\text { Other Facility Construction } \\
\text { Activities }\end{array}$ & $\begin{array}{l}\text { Not initiated due to work } \\
\text { stoppage }\end{array}$ \\
\hline \multirow{3}{*}{$\begin{array}{l}3.5 \text { Full Scale Integrated System } \\
\text { Testing }\end{array}$} & Test Plan & Completed \\
\hline & Startup and Shakedown & $\begin{array}{l}\text { Not initiated due to work } \\
\text { stoppage }\end{array}$ \\
\hline & 30 Day Test & $\begin{array}{l}\text { Not initiated due to work } \\
\text { stoppage }\end{array}$ \\
\hline
\end{tabular}

Note: Scope items for Phase 3 based on contract modification A018, dated June 1996.

\section{Task 3.1 Test System Design Criteria Definition}

Activities included under this task included the development of design criteria for an integrated pretreatment/vitrification plant, development of the operational plan, preparation of an Operational Health and Safety Plan and preparation of a Quality Assurance Plan.

\section{$\underline{\text { Design Criteria for Integrated Pretreatment/Vitrification Plant }}$}

The design criteria for the integrated pretreatment/vitrification plant were completed. Primary criteria included the requirement to process drummed waste containing contaminated soils, mud, concrete rubble up to 1 foot length, rebar up to 1 inch in diameter. Sampling of the drum is done without the need for manual removal of the drum lids. The moisture content of the drummed waste was specified to by up to $30 \%$, which for mud is the consistency of peanut butter. The maximum size steel drum to be processed is 85 gallons. The drums are shredded, and the waste dried to less than $5 \%$ moisture and homogenized so as to allow automatic sampling during delivery to an ST- 
90 Box. The ST-90 Box provides an equivalent volume capacity of approximately 12 each 55 gallon drums. It is the intent of the process to reduce the sampling and characterization costs by a factor of 10 relative to the sampling and characterization of individual drums. For the demonstration testing, the system will not be processing organic or mercury containing materials; however the process will provide provisions for the addition of activated carbon filter assemblies to allow for the processing of these materials in the future.

\section{Preparation of the Operational Plan}

An Operational Plan was under development in accordance with DOE Order DOE 5480.19. This Plan addresses operational procedures including the following:

- Organization of the plant personnel, including staffing plan and job descriptions

- Definition of operational practices

- Activities in controlled areas

- Communication systems

- Training procedures

- Investigations of abnormal events

- Notification procedures

- Control of equipment and system status procedures

- Lockout and tag out procedures

- Independent verification procedures

- $\quad$ Log keeping procedures

\section{Preparation of the Operational Health and Safety Manual}

A draft Operational Health and Safety Plan (HASP) initially developed by SMS (a DOE Paducah subcontractor) was completed for Phase 3 of the project.

\section{Quality Assurance Plan}

The Quality Assurance Plan for the project was issued to DOE for review in 1996 and revised in 1997 and 1998.

\section{$\underline{\text { Task 3.2 Component Design and Fabrication }}$}

The primary activities under this task included development of an engineering quality assurance plan, detailed design and procurement of the primary process components and storage/maintenance of equipment delivered to the site.

\section{Component Design/Procurement Tasks}

Design and procurement of approximately $75 \%$ of the process subsystems and equipment was completed, and materials were purchased and delivered to the PGDP site as of September 1999. 
Equipment Table 6-1 provides a list of equipment that was procured and delivered to the site under Phase 3 of this project.

Purchase orders were issued for the detailed design of belt conveyors and dust collection systems, and engineering activity continued toward finalizing those designs prior to release for fabrication. Preliminary specifications for the wastewater treatment system were completed and quotes were being evaluated prior to order placement. Other in-process design efforts included electrical, instrumentation and controls finalization, front-end structural steel design finalization, and the completion of a specification and procurement package for soliciting construction/installation bids.

\section{Ongoing Maintenance and Storage Effort}

Based on DOE's consent agreement, no assembly of equipment was to take place at the site until the EA was completed and a FONSI issued. Since May 1, 1997, provisions have been made to protect and maintain the equipment stored at the site. Approximately \$6 million worth of equipment are stored at several locations in the Paducah Area. Heavy equipment; such as silos, steel for the CMS $®$ tower, and the dryer are stored at the site. Smaller equipment is stored in the clamshell shelter adjacent to the site and in several temporary containers on the site. Equipment that would be adversely affected by extreme temperatures or humidity, is being stored at a commercial warehouse in Paducah

Vortec continues to conduct a maintenance program for the equipment. This equipment is inspected once a month and, to the extent feasible, its condition is determined. Repairs, such as painting of rust spots and the maintaining of the temporary structures are made on an as-needed basis.

In late 2000, Vortec was instructed to place all equipment not to be used during Phase 3A on a surplus list for disposition. As of the date of this report, only one item of equipment, the Wastewater Loading Rack, has been transferred to another owner. All equipment is to be maintained until installed or disposed.

\section{$\underline{\text { Task 3.3 Integrated System Design }}$}

Vortec designed the mechanical interfaces between the feed preparation, batch and blend, vitrification, product handling, air pollution and wastewater treatment systems. Purchase orders were issued for the detailed design of belt conveyors and dust collection systems, and engineering activity continued toward finalizing designs so those interface equipments could be released for fabrication. Structural towers for mounting shredders, dust collection, and conveyors were designed and ready for detailing upon completion of shredder and dust collection designs.

\section{Task 3.4 Integrated System Facility Construction}

During the years 1996 and 1997, Vortec initiated construction. Site preparation work was completed including excavation of unconsolidated fill materials, placement of geotextiles, and construction of concrete pads with anchor bolts for equipment mounting. Under the 1997, consent agreement DOE 
agreed to discontinue site work and not to commence facility construction while they prepared the Environmental Assessment.

\section{$\underline{\text { Task 3.5 Integrated System Testing }}$}

A draft Test Plan was developed and submitted for review. As part of the negotiations with the Kentucky Department of Waste Management, a Demonstration Test Plan (DTP) in accordance with EPA protocol was being prepared through an EPA subcontractor. The DTP was based on Vortec's Test Plan and was required as part of the RD\&D permit application. The DTP included a sampling and analytical QAPP and the sampling protocol. A draft test plan was prepared in early 1998 and distributed to the project team and the State for comments. As of early 2000, the EPA Site Program had partially completed the final DTP document. EPA withdrew their support for the demonstration test plan when DOE decided to discontinue the vitrification portion of the program. 
Vortec Corporation

NETL Phase 3 Final Report July 12, 2001

Page No. 18

\section{Table 4.2.1.1-1 Phase 3 Purchased Equipment}

\section{Equipment Title}

Gateway Computer

Gateway Computer

Printers

Dry Air Receiver

Product Weight Scale

Product Weight Scale

CMS $^{\mathrm{TM}}$ Combustion Air Blower

Combustion Air Filter

Compressed Air Skid Washer

Compressed Air Skid Bushing

Compressed Air Skid Washer

Compressed Air Skid Automatic Drain

Compressed Air Skid Safety Valve

Compressed Air Skid

Compressed Air Skid Locking Plate

Air Compressor Cooling Fan

Compressed Air Skid Ball Valve

Compressed Air Skid Bushing

Compressed Air System Control Panel

Compressed Air Skid Bolt

Compressed Air Skid Cap Screw

Compressed Air Skid Nut

Compressed Air Skid Parallel Key

Compressed Air Skid Parallel Key

Compressed Air Skid Ball Valve

Compressed Air Skid Adapter

Compressed Air Skid

Compressed Air Skid Bushing

Air Dryer Particulate Filter

Air Dryer

Air Compressor Oil Filter

Air Compressor Oil Separator

Air Compressor Moisture Separator

Air Dryer Coalescing Filter

Air Dryer Inlet Air Silencer 1

Compressed Air Skid Roller Bearing

Air Dryer Inlet Air Silencer 2

Compressed Air Skid Drive Shaft

Air Dryer Local Control Panel

Compressed Air Skid

Compressed Air Skid Roller Bearing

Compressed Air Skid Seal Washer

\section{Date Dlvrd}

$7 / 12 / 96$

$7 / 12 / 96$

$7 / 27 / 96$

$8 / 28 / 96$

$10 / 16 / 96$

$10 / 21 / 96$

$10 / 21 / 96$

$12 / 10 / 96$

$12 / 10 / 96$

$12 / 20 / 96$

$12 / 20 / 96$

$12 / 20 / 96$

$12 / 20 / 96$

$12 / 20 / 96$

$12 / 20 / 96$

$12 / 20 / 96$

$12 / 20 / 96$

$12 / 20 / 96$

$12 / 20 / 96$

$12 / 20 / 96$

$12 / 20 / 96$

$12 / 20 / 96$

$12 / 20 / 96$

$12 / 20 / 96$

$2 / 20 / 96$

$12 / 20 / 96$

$12 / 20 / 96$

$12 / 20 / 96$

$12 / 20 / 96$

$12 / 20 / 96$

$12 / 20 / 96$

$12 / 20 / 96$

$12 / 20 / 96$

$12 / 20 / 96$

$12 / 20 / 96$

$12 / 20 / 96$

$12 / 20 / 96$

$12 / 20 / 96$

$12 / 20 / 96$

$12 / 20 / 96$

$12 / 20 / 96$

$12 / 20 / 96$

$12 / 20 / 96$

\section{Equipment Title}

Cooling System Reservoir Pump

Cooling Tower Spray Pump

Cooling Tower Local Control Panel

Cooling Water Pump

Cooling Tower Ski

Cooling Tower

Cooling System Reservoir Tank

Cooling Water Pump

Cooling Tower Fan

Cooling Tower Fan

Cooling Tower Fan

Cooling System Surge Tank

Cooling System Surge Tank

Process Offgas Heater Skid Temp. Controller

Process Off Gas Exhaust Stack Exp. Joint

FM-IRI Gas Controller

Process Offgas Heater Skid Temp. Controller

Process Offgas Heater Skid

Process ID Blower Outlet Exp. Joint

Process ID Blower Inlet Exp. Joint

Process Off Gas Heater Outlet Duct Exp. Join

Process Off Gas Heater Inlet Duct Exp. Joint

Process Offgas HEPA

Process Offgas HEPA

Process Offgas Heater

Process ID Blower

Crusher Crate Components

Crusher

Scalping Screen Fines Outlet Flex. Connection

Scalping Screen Course Outlet Flex. Connection

Scalping Screen Inlet Flexible Connection

Finishing Screen

Intermediate Screen Support Leg

Intermediate Screen V-Belt

Intermediate Screen Bushing

Intermediate Screen Motor Base

Scalping Screen Motor

Intermediate Screen

Scalping Screen Support Leg

Intermediate Screen Inlet Flex. Connection

Scalping Screen Bushing

Intermediate Screen Motor Pulley

Finishing Screen End Clamp

\section{Date Dlvrd}

$1 / 10 / 97$

$1 / 10 / 97$

$1 / 10 / 97$

$1 / 10 / 97$

$1 / 10 / 97$

$1 / 10 / 97$

$1 / 10 / 97$

$1 / 10 / 97$

$1 / 10 / 97$

$1 / 10 / 97$

$1 / 10 / 97$

$1 / 10 / 97$

$1 / 23 / 97$

$1 / 23 / 97$

$1 / 23 / 97$

$1 / 23 / 97$

$1 / 23 / 97$

$1 / 23 / 97$

$1 / 23 / 97$

$1 / 23 / 97$

1/23/97

$1 / 23 / 97$

$1 / 23 / 97$

$1 / 23 / 97$

$2 / 3 / 97$

2/3/97

$2 / 24 / 97$

$2 / 24 / 97$

$2 / 24 / 97$

$2 / 24 / 97$

$2 / 24 / 97$

$2 / 24 / 97$

2/24/97

$2 / 24 / 97$

$2 / 24 / 97$

$2 / 24 / 97$

$2 / 24 / 97$

2/24/97

$2 / 24 / 97$

$2 / 24 / 97$

2/24/97

\section{Equipment Title}

Finishing Screen Vibrating Side Clamp

Scalping Screen Motor Pulley

Finishing Screen Main Frame Side Clamp

Scalping Screen Motor Base

Scalping Screen

Scalping Screen V-Belt

Finishing Screen Fine Outlet Flex. Connection

Intermediate Screen Moto

Finishing Screen Course Outlet Flex. Connection

Intermediate Screen Fines Outlet Flex. Conn.

Finishing Screen Inlet Flexible Connection

Finishing Screen Fines Outlet Flex. Connection

Intermediate Screen Course Outlet Flex. Conn.

Hot Gas Piping (6" dia. x 43" OAL)

Hot Gas Piping (10" dia. x 143.63" OAL)

Hot Gas Piping (10" dia. x 160.63" OAL)

Hot Gas Piping (16" Insulated Flange Cover

Hot Gas Piping (6" dia. x 20" OAL)

Hot Gas Ppg (10UT-10LP-F7-.025 SS304x101"

Combustion Heat Exch. Outlet Expansion Joint

Hot Gas Piping (Misc. Ass'y 36" OAL)

Air Heater Skid's Loose Components

Air Heater Skid

Combustion Heat Exch. Inlet Expansion Joint

Hot Gas Piping (16" Pipe Ass'y (95.57" OAL)

Hot Gas Piping (8" dia. x 111" OAL)

Hot Gas Piping

Hot Gas Piping (10" Insulated Flange Cover

Hot Gas Ppg (16UT-15LP-F7-.025 SS304x108"

Air Heater

Combustion Heat Exchanger

Air Heater Stack

Recuperator Thermocouples Type K

Recuperator Base Ring (Refractory Lined)

Recuperator Constant Load Hangers

Recuperator

Liquid Propane Tank Internal Spring Relief Valve

Liquid Propane Tank Adapter Flange

Liquid Propane Tank Gas Pressure Regulato

Liquid Propane Tank XS Flow Valve

Liquid Propane Tank XS Flow Valve

Liquid Propane Tank

Liquid Propane Tank Rain Cap

\section{Date Dlvrd}

$2 / 24 / 97$

$2 / 24 / 97$

$2 / 24 / 97$

$2 / 24 / 97$

$2 / 24 / 97$

$2 / 24 / 97$

$2 / 24 / 97$

$2 / 24 / 97$

$2 / 24 / 97$

$2 / 24 / 97$

$2 / 24 / 97$

2/24/97

2/24/97

$2 / 25 / 97$

$2 / 25 / 97$

2/25/97

$2 / 25 / 97$

/25/97

$2 / 25 / 97$

2/25/97

$2 / 25 / 97$

$2 / 25 / 97$

2/25/97

2/25/97

2/25/97

$2 / 25 / 97$

$2 / 25 / 97$

$2 / 25 / 97$

2/25/97

2/25/97

$2 / 25 / 97$

$2 / 26 / 97$

$3 / 12 / 97$

$3 / 12 / 97$

$3 / 12 / 97$

$3 / 12 / 97$

$3 / 19 / 97$

$3 / 19 / 97$

$3 / 19 / 97$

$3 / 19 / 97$

$3 / 19 / 97$

$3 / 19 / 97$

$3 / 19 / 97$ 
Vortec Corporation

NETL Phase 3 Final Report

July 12, 2001

Page No. 19

\section{Equipment Title}

Liquid Propane Tank Thermometer

Liquid Propane Tank Float Gage

Liquid Propane Tank Shut-Off \& Outage Valve

Liquid Propane Tank NTP Pipeway Adapter

Liquid Propane Tank Rotary Liquid Level Gage

Liquid Propane Tank Pressure Gage

Off Gas Damper

Nuisance Dust Collector Screw Conveyor

Dryer Dust Collector Screw Conveyor

Nuisance Rotary Air Lock

4" Dryer Dust Collector Damper

Dryer D.C. Rotary Airlock

HEPA Filter (Nuisance)

Nuisance Dust Collector Skid

Hepa Filter Outlet Flex. Connection

Dryer Dust Collector

Nuisance Dust Collector

Hepa Filter Inlet Flex. Connection

Nuisance Dust Collector Outlet Flex. Connection

HPU Cooler Fan

Secondary Drum Shredder Hydraulic Pump

Primary Drum Shredder Hydraulic Pump

Primary Shredder RAM

Feed Preparation Control Panel

Secondary Drum Shredder Pump

Primary Drum Shredder Hyd. Pump Outlet Filter

Primary Shredder RAM Hydraulic Pump

Secondary Shredder Oil Filter

Secondary Drum Shredder Oil Return Line Filter

Secondary Drum Shredder Hyd. Pump Outlet

Primary Drum Shredder Hyd. Pump Return Filter

Hydraulic Reservoir Oil Return Filter

Secondary Drum Shredder

ME-154 Hydraulic Skid

Primary Drum Shredder

ME-101 Hydraulic Skid

Hydraulic Reservoir

Hydraulic Reservoir

Primary Drum Shredder Fan

Air Compressor

\section{Date Dlvrd}

$3 / 19 / 97$

$3 / 19 / 97$

$3 / 19 / 97$

$3 / 19 / 97$

$3 / 19 / 97$

$3 / 19 / 97$

$3 / 24 / 97$

$3 / 24 / 97$

$3 / 24 / 97$

$3 / 24 / 97$

$3 / 24 / 97$

$3 / 24 / 97$

$3 / 24 / 97$

$3 / 24 / 97$

$3 / 25 / 97$

$3 / 25 / 97$

$3 / 25 / 97$

$3 / 25 / 97$

$3 / 25 / 97$

$3 / 31 / 97$

$3 / 31 / 97$

$3 / 31 / 97$

$3 / 31 / 97$

$3 / 31 / 97$

$3 / 31 / 97$

$3 / 31 / 97$

$3 / 31 / 97$

$3 / 31 / 97$

$3 / 31 / 97$

$3 / 31 / 97$

$3 / 31 / 97$

$3 / 31 / 97$

$3 / 31 / 97$

$3 / 31 / 97$

$3 / 31 / 97$

$3 / 31 / 97$

$3 / 31 / 97$

$3 / 31 / 97$

$3 / 31 / 97$

$4 / 18 / 97$

\section{Equipment Title}

WESP Purge Blower Inlet Air Filter

WESP Purge Blower Flex. Connection

WESP Blowdown Pump Outlet Flex. Connection

WESP Blowdown Pump Inlet Flex. Connection

WESP Irrigation Chamber 18" FRP Saddle

Venturi Recycle Pump Outlet Flex. Connection

Venturi Recycle Pump Inlet Flex. Connection

Limestone Storage Silo

WESP Irrigation Chamber 21" FRP Saddle

Separator Vessel WESP Inlet Duct

WESP Inlet Duct Expansion Joint

WESP Outlet Duct Expansion Joint

Separator Vessel Outlet Duct

Soda Ash Storage Silo

Soda Ash Skid

Separator Outlet Duct Expansion Joint

WESP Irrigation Chamber

Limestone Storage Skid

Magnet Head Pulley

Tramp Iron Magne

Metal Detector

Tramp Iron Magnet Rectifie

Waterbath Recirculation Path Pump

Propane Vaporizer Skid

Propane Tank Pump

Propane Vaporizer Skid Angle Globe Valve

Propane Pumping Skid Hydrostatic Relief Valve

Propane Vaporizer

Propane Vaporizer Control Pane

Propane Pumping Skid Mounting Clips

Propane Truck Unloading Skic

Propane Vaporizer Skid Angle Globe Valve

Glass Quench Tank Skid

Glass Quench Heat Exchanger

Glass Quench Surge Tank

Glass Quench Tank 4" Gate Valves

Quench Water Circulation Pump

Cooling Water Rack Skid

Glass Quench Control Panel

Cooling Water Hydraulic Hose Tray

\section{Date Dlvrd}

$4 / 29 / 97$

$4 / 29 / 97$

$4 / 29 / 97$

$4 / 29 / 97$

$4 / 29 / 97$

$4 / 29 / 97$

$4 / 29 / 97$

$4 / 29 / 97$

$4 / 29 / 97$

$4 / 29 / 97$

$4 / 29 / 97$

$4 / 29 / 97$

$4 / 29 / 97$

$4 / 29 / 97$

$4 / 29 / 97$

$4 / 29 / 97$

$4 / 29 / 97$

$4 / 29 / 97$

$5 / 6 / 97$

$5 / 6 / 97$

$5 / 6 / 97$

$5 / 6 / 97$

$5 / 8 / 97$

$5 / 8 / 97$

$5 / 8 / 97$

$5 / 8 / 97$

$5 / 8 / 97$

$5 / 8 / 97$

$5 / 8 / 97$

$5 / 8 / 97$

$5 / 8 / 97$

$5 / 8 / 97$

$5 / 12 / 97$

$5 / 12 / 97$

$5 / 12 / 97$

$5 / 12 / 97$

$5 / 12 / 97$

$5 / 12 / 97$

$5 / 12 / 97$

$5 / 12 / 97$

\section{Equipment Title}

VSI Mill Lube Pump

VSI Mill Lid Lifter Pump

Emergency Diesel Generator Tail Pipe

Emergency Diesel Generator Muffle

Emergency Diesel Generator Instruction Books

Emergency Diesel Generator Skid

Emergency Diesel Generator Battery Boxes

Emergency Diesel Generator Exhaust Gaskets

Emergency Diesel Generator Spring Type Vibs

Emergency Diesel Generator Exhaust Flex

Emergency Diesel Generator Muffler Mtg.

Emergency Diesel Generator Batteries

Emergency Diesel Generator

Emergency Diesel Generator Pad Type Vib

WESP Trans/Rectifier Control Pane

WESP T/R Skid high volt. Pipe \& Guard Adder

Wet Electrostatic Precipitator

WESP T/R Skid

WESP T/R Skid High Volt. Pipe \& Guard

WESP T/R Skid High Volt. Pipe \& Guard

Blending System Miscellaneous Parts

Batch Transporter Containment Pipe Bends Skid

Batch Transporter Containment Pipe Flex Conne

Batch Blending Bin

Containment Pipe Manual Ball Valve Skid

Feedstock Transporter

Feedstock Transporter Hardy Weigh Chassis

Batch Transporter Containment Pipe Skid

Air Receiver

Batch Dust Collector

Bottom Transporter

Top Transporter

Feedstock Transition Hopper

Limestone Dust Collecto

Feedstock Dust Collector

Batch Volumetric Feeder

Limestone Feeder

Batch Transporter Containment Pipe Skid

Soil Feeder

Soda Ash Feeder

\section{Date Dlvrd}

$5 / 13 / 97$

$5 / 13 / 97$

$5 / 20 / 97$

$5 / 20 / 97$

$5 / 20 / 97$

$5 / 20 / 97$

$5 / 20 / 97$

$5 / 20 / 97$

$5 / 20 / 97$

$5 / 20 / 97$

$5 / 20 / 97$

$5 / 20 / 97$

$5 / 20 / 97$

$5 / 20 / 97$

$5 / 28 / 97$

$5 / 28 / 97$

$5 / 28 / 97$

$5 / 28 / 97$

$5 / 28 / 97$

$5 / 28 / 97$

$6 / 11 / 97$

$6 / 11 / 97$

$6 / 11 / 97$

$6 / 11 / 97$

$6 / 11 / 97$

$6 / 11 / 97$

$6 / 11 / 97$

$6 / 11 / 97$

$6 / 11 / 97$

$6 / 11 / 97$

$6 / 11 / 97$

$6 / 11 / 97$

$6 / 11 / 97$

$6 / 11 / 97$

$6 / 11 / 97$

$6 / 11 / 97$

$6 / 11 / 97$

$6 / 11 / 97$

$6 / 11 / 97$

$6 / 11 / 97$ 
Vortec Corporation

NETL Phase 3 Final Report July 12, 2001

Page No. 20

PLC Equipment

Separator

Irrigation Chamber Skid

$4 / 22 / 97$

$4 / 29 / 97$

$4 / 29 / 97$

\section{Equipment Title}

CRV Seismic Restraints

CRV Seismic Restraints

CMS $^{\text {TM }}$ Tower Steel Bracing

CMSTM Tower Steel

CMS $^{\text {TM }}$ Tower Steel Stair Treads

CMS $^{\text {TM }}$ Tower Steel Columns

CMS TM Tower Steel Beams

Dryer Discharge Airlock Gate B

Dryer Feed Airlock Gate A

Dryer Feed Airlock Gate B

Dryer Discharge Airlock Gate A

Cooling Tower Fan

Vapor Recovery Skid

Vapor Recovery Unit HX

Vapor Recovery Recirculating Pump

Vapor Recovery Condenser Pump

Vapor Recovery HX Skid

Vapor Recovery HX Skid Interconnecting

Vapor Recovery HX Skid Flex. Inlet Duct Pallet

Vapor Recovery HX Skid Piping \& Spool Pieces

Vapor Recovery HX Skid (Cartons of Packing)

Vapor Recovery HX Skid (Crate of Instruments)

Vapor Recovery Condenser

Vapor Recovery Cooling Tower

CMSTM Refractory (fired Shapes)

Cyclone Melter Inlet End Spool

Cyclone Melter Expansion Controllers

Recuperator Base 2

Recuperator Base

Recuperator Base Inlet/Item M-2

Recuperator Base Inlet/Item M-3

Recuperator Base Blind Flange

Recuperator Base Plate Blind Flange

Cyclone Melter Outlet End Flange

Cyclone Melter Outlet End Spool
Glass Quench Tank Loose Parts

VSI Mill

VSI Mill Inlet Flex. Connection
$5 / 12 / 97$

$5 / 13 / 97$

$5 / 13 / 97$
Soda Ash Dust Collector

Batch Blending Skid

Batch Transporter Skid
$6 / 11 / 97$

$6 / 11 / 97$

$6 / 11 / 97$

\section{Date Dlvrd}

$6 / 20 / 97$

$6 / 20 / 97$

$6 / 30 / 97$

$6 / 30 / 97$

$6 / 30 / 97$

$6 / 30 / 97$

$6 / 30 / 97$

$7 / 11 / 97$

$7 / 11 / 97$

$7 / 11 / 97$

$7 / 11 / 97$

$8 / 26 / 97$

$8 / 26 / 97$

$8 / 26 / 97$

$8 / 26 / 97$

$8 / 26 / 97$

$8 / 26 / 97$

$8 / 26 / 97$

$8 / 26 / 97$

$8 / 26 / 97$

$8 / 26 / 97$

$8 / 26 / 97$

$8 / 26 / 97$

$8 / 26 / 97$

9/5/97

9/10/97

9/10/97

$9 / 10 / 97$

$9 / 10 / 97$

9/10/97

$9 / 10 / 97$

$9 / 10 / 97$

$9 / 10 / 97$

9/10/97

9/10/97
CMS $^{\mathrm{TM}}$ Recuperator Plenum MK Rnd. Blt.

CMS $^{\text {TM }}$ Single Tab Ceramic Pillow

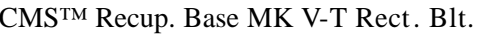

CMSTM Double Tab Ceramic Pillow

CMS $^{\text {TM }}$ Bundle Bars/Fasteners

CMS $^{\text {TM }}$ Single Tab Pillow

CMS $^{\text {TM }}$ Fabric Expansion Joints

Air Heater Fan

Air Heater Fan Inlet Vein Damper

Air Heater Fan Silencer Support

Air Heater Fan Silencer

WESP Skid Galvanized Pipe Sections

WESP Blowdown Pump

WESP Skid

WESP Skid Pollution Ground Sets Cartons

WESP Skid Katron RF Point Level Switch

WESP Skid Lapp Insulators

Quencher/Venturi Scrubber

WESP Skid Crated Piping

WESP Skid Venturi w/Lined Inlet

WESP Skid 1' 6" Dia. Expansion Joint

WESP Skid 1' 9" Dia. Expansion Joint

WESP Purge Blower

WESP Blowdown Tank

WESP Skid Magnetrol level detector

Venturi Scrubber Recycle Pump

CRV Heat Up Burner A

Heat Up Burner "B" Skid

CRV Flame Safety Panel

CRV Flame Safety Panel 2 Amp Fuses

CRV Flame Safety Panel Pipe Rack Junction Box

CRV Heat Up Burner B

CRV Flame Safety Panel 10 Amp Fuses

CRV Propane Control 3-Valve Manifold

CRV Propane Control Skid

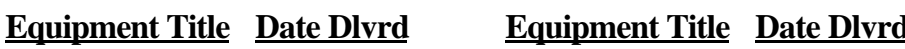

$9 / 25 / 97$

$9 / 25 / 97$

$9 / 25 / 97$

$9 / 25 / 97$

$9 / 25 / 97$

$9 / 25 / 97$

$9 / 25 / 97$

$10 / 29 / 97$

$10 / 29 / 97$

$10 / 29 / 97$

$10 / 29 / 97$

$11 / 13 / 97$

$11 / 13 / 97$

$11 / 13 / 97$

$11 / 13 / 97$

$11 / 13 / 97$

$11 / 13 / 97$

$11 / 13 / 97$

$1 / 13 / 97$

$11 / 13 / 97$

$11 / 13 / 97$

$11 / 13 / 97$

$11 / 13 / 97$

$11 / 13 / 97$

$11 / 13 / 97$

$11 / 13 / 97$

$1 / 13 / 98$

$1 / 13 / 98$

$1 / 13 / 98$

$1 / 13 / 98$

$1 / 13 / 98$

$1 / 13 / 98$

$1 / 13 / 98$

$1 / 13 / 98$

$1 / 13 / 98$
RAS Diffusor Fan 45 deg. Elbow w/Straight Duct

RAS Drum Screen

RAS Diffusor Fan Straight duct (DQ-104)

RAS Diffusor Fan Transition (DQ-107)

RAS Cyclone Foot (DQ-122-1,2,3,4)

RAS Cyclone Tower (DQ-122)

RAS Cyclone

Air Knife, Fine Material

Air Knife, Coarse Material

RAS Cyclone Fan Horiz. Duct (DQ-110)

RAS Cyclone Fan Vert. Duct w/45 deg. Elbow

RAS Diffusor Fan

Coarse Air Knife Ducting (DQ-201)

Fine Air Knife Blower Ducting (DQ-301)

RAS Belt Feed

RAS Cyclone Fan

RAS Diffusor Fan Straight Duct (DQ-106)

Coarse Air Knife Blower

RAS Cyclone Fan Transition (DQ-109)

RAS Cyclone Fan Horiz. Duct w/90 deg. Elbow

Product Weight Scale Strain Gage Transmitter

Drum Scale

CMS ${ }^{\text {TM }}$ O-Rings

CMS ${ }^{\text {TM }}$ View Ports

CMSTM Spring Hanger Ass'y "H-6"

CMSTM Spring Hanger Ass'y "H-2"

CMS $^{\text {TM }}$ Spring Hanger Ass'y "H-10"

$\mathrm{CMS}^{\mathrm{TM}}$ Spring Hanger Ass'y "H-11"

CMS TM Spring Hanger Ass'y "H-12"

CMS $^{\text {TM }}$ Spring Hangers

$\mathrm{CMS}^{\mathrm{TM}}$ Spring Hanger Ass'y "H-13"

CMSTM Spring Hanger Ass'y "H-1"

CMSTM Spring Hanger Ass'y "H-3"

CMSTM Spring Hanger Ass'y "H-5"

CMS ${ }^{\text {TM }}$ Spring Hanger Ass'y "H-7"
$5 / 20 / 98$

$5 / 20 / 98$

$5 / 20 / 98$

$5 / 20 / 98$

$5 / 20 / 98$

$5 / 20 / 98$

$5 / 20 / 98$

$5 / 20 / 98$

$5 / 20 / 98$

$5 / 20 / 98$

$5 / 20 / 98$

$5 / 20 / 98$

$5 / 20 / 98$

$5 / 20 / 98$

$5 / 20 / 98$

$5 / 20 / 98$

$5 / 20 / 98$

$5 / 20 / 98$

$5 / 20 / 98$

$5 / 20 / 98$

$8 / 27 / 98$

$8 / 27 / 98$

$9 / 1 / 98$

9/22/98

9/29/98

9/29/98

$9 / 29 / 98$

9/29/98

$9 / 29 / 98$

9/29/98

9/29/98

9/29/98

9/29/98

9/29/98

$9 / 29 / 98$ 
Cyclone Melter

CRV Combustor

Cyclone Melter Inlet End Flange

Dryer Dust Collector Fan

Nuisance Dust Collector Damper

Dryer Dust Collector Damper

Nuisance Dust Collector Fan

CMS $^{\text {TM }}$ Double Tab Ceramic Pillow
9/10/97

9/10/97

$9 / 10 / 97$

9/23/97

9/23/97

$9 / 23 / 97$

9/23/97

$9 / 25 / 97$

Date Dlvrd

$10 / 7 / 98$

$10 / 7 / 98$

$10 / 7 / 98$

$10 / 7 / 98$

$10 / 7 / 98$

$10 / 7 / 98$

$10 / 7 / 98$

$10 / 7 / 98$

$10 / 7 / 98$

$10 / 7 / 98$

$10 / 14 / 98$

$11 / 4 / 98$

$11 / 4 / 98$

$11 / 4 / 98$

$11 / 4 / 98$

$11 / 4 / 98$

$11 / 4 / 98$

$1 / 4 / 98$

$11 / 4 / 98$

$11 / 4 / 98$

$11 / 4 / 98$

$11 / 5 / 98$

$11 / 5 / 98$

$11 / 5 / 98$

$11 / 5 / 98$

$11 / 5 / 98$

$11 / 5 / 98$

$11 / 24 / 98$

$11 / 24 / 98$

$11 / 24 / 98$

$11 / 24 / 98$

$1 / 24 / 98$

$1 / 24 / 98$
CRV Flame Safety Panel 5 Amp Fuses

Heat Up Burner "A" Skid

Soil Storage Silo

Soil Storage Skid

High Airflow Rotary Dryer Drum

High Airflow Rotary Dryer

RAS Rotary Air Lock

Rotary Air Separator Skid
$1 / 13 / 98$

$1 / 13 / 98$

$2 / 12 / 98$

$2 / 12 / 98$

$4 / 8 / 98$

$4 / 8 / 98$

$5 / 20 / 98$

$5 / 20 / 98$

Vortec Corporation

NETL Phase 3 Final Report July 12, 2001

Page No. 21

$9 / 29 / 98$

$9 / 29 / 98$

$9 / 29 / 98$

$10 / 7 / 98$

$10 / 7 / 98$

$10 / 7 / 98$

$10 / 7 / 98$

$10 / 7 / 98$

\section{Equipment Title}

Industrial Shop Vacuum Cleaner Floor Lance

Industrial Shop Vacuum Cleaner Hose

Industrial Shop Vacuum Cleaner Small

Glass Conveyor Discharge Gate

Drag Conveyor Steel Knee Braces

Gas Seal Chute

Drag Conveyor Miscellaneous Parts

Drag Conveyor Chain Guard

Drag Conveyor Steel Duct Reducers

Glass Quench Conveyor Skid

Gas Seal Chute Hand Chain Hoist

ST-90 Pneumatic Hood 3/8" x 4" Gasket Roll

Drag Conveyor Round Steel Chutes

Drag Conveyor Steel Legs

Drag Conveyor Chain

Drag Conveyor Flights

Drag Conveyor Motor \& Gear Reducer

Drag Conveyor Tail \& Trough Section

Glass Quench Drag Conveyor

Drag Conveyor Discharge Valve

ST-90 Pneumatic Hood Flex Hose

ST-90 Pneumatic Hood 2" Pipe U-Bolts

ST-90 Pneumatic Hood 1" Pipe U-Bolts

ST-90 Pneumatic Hood Adj. Hose Clamp

Drag Conveyor Steel "T" Top

ST-90 Pneumatic Hood Vinyl Cuff

ST-90 Pneumatic Hood 3/8" Pipe U-Bolts

Cyclone Screw Conv. (C-9) Speed Switch

Cyclone Screw Conveyor (C-9)

Inclined Screw Conv. (C8) Head Section

Cyclone Screw Conv. (C-9) Limit switch

Inclined Screw Conv. (C8) Head Section

Inclined Screw Conv. (C8) Speed Switch
Date Dlvrd

$12 / 18 / 98$

$12 / 18 / 98$

$12 / 18 / 98$

$2 / 2 / 99$

$2 / 15 / 99$

$2 / 15 / 99$

$2 / 15 / 99$

$2 / 15 / 99$

$2 / 15 / 99$

$2 / 15 / 99$

$2 / 15 / 99$

$2 / 15 / 99$

$2 / 15 / 99$

$2 / 15 / 99$

$2 / 15 / 99$

$2 / 15 / 99$

$2 / 15 / 99$

$2 / 15 / 99$

$2 / 15 / 99$

$2 / 15 / 99$

$2 / 15 / 99$

$2 / 15 / 99$

$2 / 15 / 99$

$2 / 15 / 99$

$2 / 15 / 99$

$2 / 15 / 99$

$2 / 15 / 99$

$3 / 8 / 99$

$3 / 8 / 99$

$3 / 8 / 99$

$3 / 8 / 99$

$3 / 8 / 99$

\section{Equipment Title}

Skip Hoist Bucket Assembly

Glass Channel Heated Drain Hole SCR

Glass Channel Heated Drain Hole (3/4")

Glass Channel Heated Drain Hole (1/2")

Glass Channel Propane Control Skid

Glass Channel Comb. Sys. Blower

Glass Channel Comb. Sys. Pipe Rack

Glass Channel Comb. Air. Filter

Glass Channel Comb. Sys. Burne

Glass Channel Comb. Sys. Burner

Glass Channel Comb. Sys. Burne

Glass Channel Cor

Glass Channel Comb. Sys. Burne

Glass Channel Flame Safety Pane

Glass Channel Comb. Sys. Burner

Pneumatic Product Air Lock

Pneumatic Product Air Lock

VSI Mill Pneumatic Airlock

VSI Mill Pneumatic Airlock

Pneumatic Conveyor Blower Acoustical

Pneumatic Blower Outlet Flex. Connection

Pneumatic Conveyor Blowe

Finishing Rotary Airlock

Pneumatic Convey. Air Filter Receiver

Wastewater Flocculation Tank

CMS ${ }^{\mathrm{TM}}$ Refractory (balance)

Course Air Knife Blower Damper

Fine Air Knife Blower Damper

Rotary Dryer Drum Insulation

Rotary Dryer Discharge End Hood Ass'y

Separator Reservoir

Rotary Dryer Ladder and Walkway

Rotary Dryer Stairs
Date Dlvrd

$3 / 19 / 99$

$3 / 31 / 99$

$3 / 31 / 99$

$3 / 31 / 99$

$4 / 5 / 99$

$4 / 5 / 99$

$4 / 5 / 99$

$4 / 5 / 99$

$4 / 5 / 99$

$4 / 5 / 99$

$4 / 5 / 99$

$4 / 5 / 99$

$4 / 5 / 99$

$4 / 5 / 99$

$4 / 13 / 99$

$4 / 13 / 99$

$4 / 13 / 99$

$5 / 6 / 99$

$5 / 7 / 99$

$5 / 7 / 99$

$5 / 7 / 99$

$5 / 11 / 99$

$5 / 16 / 99$

$5 / 17 / 99$

$5 / 20 / 99$

$5 / 20 / 99$

$5 / 21 / 99$

$5 / 21 / 99$

$5 / 21 / 99$

$5 / 21 / 99$

$5 / 21 / 99$ 
Fabric Structure Square Base Sections

Fabric Structure End Brace Sections

Fabric Structure Round Pull Bars

Fabric Structure Fabric Vinyl

Fabric Structure

High Airflow Rotary Dryer Frame

Fabric Structure Beams

Recuperator Plenum

Industrial Shop Vacuum Cleaner Wheel Floor

Industrial Shop Vacuum Cleaner Crevice Tool

Rotary Dryer Discharge End Seal Ass'y

Rotary Dryer Feed Seal Assembly

Rotary Dryer Expansion Folds
$11 / 24 / 98$

$11 / 24 / 98$

$11 / 24 / 98$

$11 / 24 / 98$

$11 / 24 / 98$

$11 / 24 / 98$

$11 / 24 / 98$

$12 / 18 / 98$

$12 / 18 / 98$

$12 / 18 / 98$

9/22/99

$9 / 22 / 99$

$10 / 6 / 99$
Inclined Screw Conv. (C8) Limit switch

Inclined Screw Conv. (C8) Gasket

clined Screw Conveyor (C8)

Skip Hoist Limit Switches

Skip Hoist Parts Skid

Skip Hoist Electrical Panels on Mounting Frame

Skip Hoist Winder Assembly

Skip Hoist Tower Guards

Skip Hoist Bucket Stop w/Oak Log

Skip Hoist
Vortec Corporation

NETL Phase 3 Final Report

July 12, 2001

Page No. 22

$5 / 21 / 99$

$5 / 21 / 99$

$6 / 2 / 99$

$7 / 31 / 99$

$7 / 31 / 99$

$7 / 31 / 99$

$7 / 31 / 99$

$7 / 31 / 99$

$7 / 31 / 99$

Rotary Dryer Feed End Dust Leg Chute

Rotary Dryer Sprocket Guard

Rotary Dryer Sprocket Segments

Rotary Dryer Sprocket Chai

Rotary Dryer Radial Station Trunnion.

Rotary Dryer Thrust Station Trunnion. 


\subsubsection{Phase 3A - Limited Demonstration}

The scope of Phase 3A refocused the project activities from efforts relating to the integrated pretreatment/vitrification process to the design, construction and demonstration of the material handling and conditioning system, plus the utilities required supporting that specific equipment.

The material handling and conditioning system consists of a portion of the feed preparation system for the full-scale integrated pretreatment/vitrification plant; for this reason, this phase of work is referred to as the "Limited Demonstration." The system to be installed and demonstrated under this scope of work is as shown on the Process Flow Diagram in Figure 4.2.2.2-1 (see ensuing section). The material handling and conditioning system facility will consist of a drum weighing and loading assembly, shredding assembly, drying process, ferrous metals separation assembly, prepared waste delivery/loading assembly, a comprehensive fugitive dust control system, the propane fuel subsystem, the compressed air system, and PLC-based controls and power distribution. The limited demonstration plant is located outside the security fence at Paducah and located on approximately five acres of land provided by the site. Approximately a third of the land set aside for the vitrification plant will be used for soil preparation and conditioning subsystem.

\subsubsection{Task Summaries}

The work (accomplished and pending) is summarized in Table 4.2.2.1-1, and status for each task is detailed in the subsequent subsections of this report. Estimated level of completion for Phase 3A work under Modification A040 (May 2000) is shown. All work on Phase 3 task items was suspended after May 2000 because DOE redirected work on Limited Demonstration tasks only. A cost proposal covering Modification A040 was submitted in June 2000, but the scope of work was not definitized pending settlement of a citizen lawsuit. In August 2000, incremental funding was received to initiate redesign of the front end process to accommodate the objectives of the limited demonstration program. The status below reflects the progress of the Limited Demonstration Activities through May 2001. 
Table 4.2.2.1-1 Phase 3A - Summary Status

\begin{tabular}{|c|c|c|}
\hline Task & Task Scope/Deliverable & Status/Remarks \\
\hline \multirow[t]{4}{*}{$\begin{array}{ll}3.1 & \text { Test System Design Criteria } \\
\end{array}$} & Update of Criteria from Phase 3 & Completed \\
\hline & Operational Plan & Revisions $75 \%$ complete \\
\hline & $\begin{array}{l}\text { Operational Health and Safety } \\
\text { Plan }\end{array}$ & Revisions $80 \%$ complete \\
\hline & Quality Assurance Plan & Revisions $80 \%$ complete \\
\hline \multirow[t]{2}{*}{$\begin{array}{l}\text { 3.2 Full Scale Component } \\
\text { Design and Fabrication }\end{array}$} & $\begin{array}{l}\text { Equipment \& Material } \\
\text { Specifications \& Drawings }\end{array}$ & Revisions $90 \%$ complete \\
\hline & Equipment Procurement & $\begin{array}{l}\text { No new equipment } \\
\text { procurements }\end{array}$ \\
\hline $\begin{array}{l}\text { 3.3 Full Scale Integrated System } \\
\text { Design }\end{array}$ & Final Design \& Documentation & Revisions $85 \%$ complete \\
\hline \multirow{2}{*}{$\begin{array}{l}\text { 3.4 Full Scale Integrated } \\
\text { Construction }\end{array}$} & Site Preparation Activities & On hold \\
\hline & $\begin{array}{l}\text { Other Facility Construction } \\
\text { Activities }\end{array}$ & $\begin{array}{l}\text { Not initiated due to work } \\
\text { stoppage }\end{array}$ \\
\hline \multirow[t]{3}{*}{$\begin{array}{l}3.5 \text { Full Scale Integrated System } \\
\text { Testing }\end{array}$} & Test Plan & $\begin{array}{l}\text { Draft revision submitted to } \\
\text { DOE }\end{array}$ \\
\hline & Startup and Shakedown & $\begin{array}{l}\text { Not initiated due to work } \\
\text { stoppage }\end{array}$ \\
\hline & 30 Day Test & $\begin{array}{l}\text { Not initiated due to work } \\
\text { stoppage }\end{array}$ \\
\hline
\end{tabular}

Note: Scope items for Phase 3A based on contract modification A040, dated May 2000.

\section{Task 3.1 Test System Design Criteria Definition}

The design criteria for the limited demonstration plant design was updated to reflect the objectives of the limited demonstration program. The primary activities related to revisions of the Operational Plan and related documentation such as updates to operations and maintenance procedures and revisions to the Operational Health and Safety Plan.

\section{- Preparation of the Operational Plan}

The O\&M Manual includes an Operational Plan developed in accordance with DOE Order DOE 5480.19. This Plan addresses operational procedures for the following items:

- Organization of the plant personnel, including staffing plan and job descriptions

- Definition of operational practices

- Activities in controlled areas

- Communication systems 
- Training procedures

- Investigations of abnormal events

- Notification procedures

- Control of equipment and system status procedures

- Lockout and tag out procedures

- Independent verification procedures

- Log keeping procedures

Procedures for many of these items had been written during Phase 3 work. Updates of the Operational Plan to reflect the changes resulting from implementation of the Limited Demonstration were initiated. The Operational Plan will also address quality assurance for the operational phase of the demonstration project.

Finalization of the operating and maintenance procedures that will be included in the Operational Plan will to require delivery of the remaining equipment to be purchased under this scope of work. The remaining equipment to be purchased includes two belt conveyors. When the fabrication drawings and vendor O\&M manuals for this equipment are delivered, the Operational Plan can be finalized.

- Completion of Process Hazards Analysis Review

Vortec held a Process Hazards Analysis (PHA) review with IUOE representatives in December 2000. The results of the PHA review were reviewed and incorporated into the design as appropriate. The PHA review will also be used to prepare for the Readiness Review, and complete the Operational Health and Safety Manual.

\section{- Preparation of the Operational Health and Safety Plan}

The second draft of the Operational Health and Safety Plan (HASP) was completed. The O\&M Manual will be updated following conduct of the PHA review and completed for the scope of the Material Handling and Conditioning facility.

\section{- Quality Assurance Plan}

This plan was previously developed and was reviewed for updates to conform to the Phase $3 \mathrm{~A}$ scope of work.

\section{$\underline{\text { Task 3.2 Component Design and Fabrication }}$}

\section{- Complete Remaining Component Design/Procurement Tasks}

Vortec's engineering staff completed most of the design documents for Phase 3A. The process calculations, mostly involving revisions as listed below have been completed Most engineering drawings have been completed, with a few exceptions: detailed electrical and instrumentation and some layout drawings need to be updated for internal consistency. There are a number of drawings requiring updating, but the changes are mostly drafting in nature. Some design work continues to determine anchor bolt modifications, due to relocating some of the Limited Demonstration equipment on the existing pads. Procurement specifications are complete, except for a limited number of items, which remain to be purchased. A detailed engineering package was sent out to four qualified contractors for soliciting construction bids. Several design documents including a 
Control Philosophy, Instrument List, Motor List and Construction Health and Safety Plan have been completed and issued to prospective bidders.

\section{- Ongoing Maintenance and Storage Effort}

Since May 1997, provisions have been made to protect and maintain the equipment stored at the site. Approximately \$6 million worth of equipment are stored at several locations in the Paducah area. Heavy equipment such as silos, steel for the CMS $®$ tower, and the dryer are stored at the site. Smaller equipment is stored in the clamshell shelter adjacent to the site and in several temporary containers on the site. Equipment that would be adversely affected by extreme temperatures or humidity, is being stored at a commercial warehouse in Paducah.

Vortec continues to conduct a maintenance program for the equipment. This equipment is inspected once a month and to the extent feasible, its condition is determined. Repairs, such as painting of rust spots and the maintaining of the temporary structures are made on an as-needed basis. Much of the equipment currently in storage will not be installed under this scope of work. Vortec will continue storage and maintenance of the equipment not installed under this scope of work.

\section{Task 3.3 Integrated System Design}

The integrated system design for the limited demonstration plant was nearly completed under Phase 3A. The remaining engineering work involves finalization of a few drawings, support for procurement (i.e. vendor submittal review, technical issue resolution, and inspection), and completion of the plans for the testing portion of the project.

Some design rework was required to complete the Phase 3A scope. This included:

- $\quad$ The ventilation requirements for all Phase 3A systems were revised to use the one dust collector that was already delivered to the site. This change involved relocating and resizing ductwork and supports. (The Phase 3 design had two dust collection systems, north and south, and the C3 and C4 conveyors vented to both systems.)

- $\quad$ The recirculating system for the dryer hot air system was changed to a once-through system utilizing the existing dust collector and HEPA filter to minimize construction costs and improve drying performance for the Limited Demonstration. Procurement of a new stack and miscellaneous process ducting were required by this change.

- $\quad$ The existing Hardy pneumatic conveying system, originally designed to pick up dust from the dust collectors and convey it to the soil silo, was reused to convey the dust from Limited Demonstration equipment to the soil loading station. This was done to control the dust emissions from the dust collectors, rather than discharging the dust to a container, where fugitive dust may escape. This change necessitated the modification of Hardy Pneumatic System dust pick-up points. 
- $\quad$ Due to changes of code since the propane system was purchased and reduced propane flow for the Limited Demonstration, some controls were added to the scope.

- $\quad$ Much of the PLC cabinet and MCC wiring had been done previously for the full-scale vitrification plant. Some wiring was redesigned to accommodate the Limited Demonstration equipment only.

\section{Task 3.4 Integrated System Facility Construction}

A detailed engineering package was sent out to four qualified contractors for soliciting construction bids in March 2001. The bid evaluations for the selection of the site general subcontractor remain to be completed. Two contractors submitted bids for construction that were within the range projected by Vortec. Upon release of the funds to complete construction, Vortec will award the contract and complete construction of the soil preparation and conditioning subsystems.

\section{Task 3.5 Integrated System Testing}

EPA had previously agreed to provide the test plans, sampling and analytical support for the vitrification project demonstration test. Vortec had been working with EPA's contractor to develop the test plans. As previously stated, these test plans were not completed and EPA withdrew its support for this project, based on the Limited Demonstration scope of work. A new test plan was developed, specific to the Limited Demonstration and was submitted in February 2001. The Test Plan was still undergoing review at DOE upon the completion of Phase 3A. Vortec will incorporate the DOE comments into the Limited Demonstration Test Plan prior to finalizing the Plan.

\subsubsection{Phase 4 - Limited Demonstration}

The primary objectives of Phase 4 of this program are to complete the final design, engineering and fabrication of the waste handling and conditioning subsystems and to construct the subsystems at the Paducah Gaseous Diffusion Plant.

\subsubsection{Task Summaries}

Phase 4 consists of seven primary tasks that include:

Task 4.1 - Site Commitment Agreement, Schedule and Cost

Task 4.2 - Test Plan Development

Task 4.3 - Operational Plan

Task 4.4 - Final Subsystems Design

Task 4.5 - Quality Assurance Plan

Task 4.6 - Facility Disposition

Task 4.7 - Construction Subsystems (Optional Task). 
In addition to periodic reporting, the deliverables include:

Site Commitment Agreement - due within 90 days of award

Test Plan - due within 60 days of award

Operational Plan - due 60 days of award

Final Design Drawings - due 90 days of award

Quality Assurance Plan - due 90 days of award

Construction Schedule - due 30 days of construction authorization

Construction Completion - within 240 days of construction authorization.

Phase 4 was initiated May 11, 2001 and completion of the first five tasks is scheduled for completion by September 11, 2001. Table 4.2.3-1 summarizes the status of these activities as of the date of this report.

Table 4.2.3-1 Phase 4 - Summary Status

\begin{tabular}{|ll|l|l|}
\hline \multicolumn{2}{|c|}{ Task } & \multicolumn{1}{c|}{ Task Scope/Deliverable } & \multicolumn{1}{c|}{ Status/Remarks } \\
\hline 4.1 & Site Commitment Agreement & $\begin{array}{l}\text { Commitment Letter, Schedule, } \\
\text { Cost }\end{array}$ & In Progress \\
\hline 4.2 & Test Plan Develoment & Test Plan & Submitted 7/10/01 \\
\hline $4.3 \quad$ Operational Plan & Operational Plan & Submitted 7/10/01 \\
\hline $4.4 \quad$ Final Subsystems DAesign & Design Review & Completed June 14, 2001 \\
\hline & & Engineering Documentation & In progress \\
\hline 4.5 & Quality Assurance Plan & Quality Assurance Plan & In Progress \\
\hline & & Equipment Procurement & $\begin{array}{l}\text { No new equipment } \\
\text { procurements }\end{array}$ \\
\hline 4.6 & Facility Disposition & Property management reports & In Progress \\
\hline 4.7 & Subsystems Construction & Construction Schedule & Option not initiated \\
\hline & & Construction Completion & Option not initiated \\
\hline
\end{tabular}

Note: Scope items for Phase 4 based on contract modification A042, dated May 11, 2001.

A summary of the task activities is provided below.

\section{Task 4.1 - Site Commitment Agreement}

Contacts with various stakeholders at DOE Paducah were made to assess the potential of their participation and/or support for the Limited Demonstration project. Stakeholders at DOE/Paducah were initially contacted because of their previous participation in the integrated waste pretreatment/vitrification activities. Potential participants from other DOE sites including DOE/Portsmouth and DOE/Oak Ridge were also contacted to assess their potential support of the demonstration project.

Based on these contacts, it was determined that Vortec's efforts should focus on completing the demonstration at Paducah. The primary reasons for making this selection included: 
1. DOE/Paducah's previous support for the integrated pretreatment/vitrification demonstration.

2. DOE/Paducah's commitment to continue support for the Limited Demonstration.

3. A commitment from USEC for cost share participation contingent upon development of a favorable path forward for securing the necessary permits and a waste management contract which will utilize the waste handling and conditioning system.

4. A favorable response from Bechtel Jacobs to pursue a project which would target the processing of drummed legacy waste on a near-term basis.

5. The existing infrastructure at Paduah (i.e., site preparation) to accommodate the limited demonstration.

6. Expected favorable support from the Kentucky regulators for the project.

7. Resolution of the law suit between the principals of the SSAB and DOE to allow the Limited Demonstration to proceed.

Based on these development, it is anticipated the necessary site agreements can be obtained to support continuation of the Limited Demonstration to the construction phase and with eventual commercial implementation.

\section{Task 4.2 - Test Plan Development}

The test plan for the Limited Demonstration has been completed and delivered to DOE on July 10, 2001. Comments from DOE/NETL and the Mixed Waste Focus Group have been integrated into the objectives, goals, testing and analyses to be performed. A summary of the basic elements of the Test Plan for the Limited Demonstration is presented in Section 4.2.4 of this report.

\section{Task 4.3 - Operational Plan}

The operational plan has been completed and was delivered to DOE on July 10, 2001. The operational Plan included the following elements:

1. Organization of the plant personnel, including staffing plan and job descriptions

2. Definition of operational practices

3. Activities in controlled areas

4. Communication systems

5. Training procedures

6. Investigations of abnormal events

7. Notification procedures

8. Control of equipment and system status procedures

9. Lockout and tag out procedures

10. Independent verification procedures

11. Log keeping procedures

12. Operations turnover procedures

13. Timely orders to operators procedures

14. Equipment labeling procedures

15. Operating and Maintenance Plan

\section{Task 4.4 - Final Subsystems Design}


Final design of the subsystems for the limited demonstration is to be completed by August 9, 2001. Delivery of the design documentation is expected to be on schedule.

\section{Task 4.5 - Quality Assurance Plan}

The Quality Assurance Plan for the limited demonstration is to be completed by August 9, 2001. Delivery of the Quality Assurance Plan is expected to be on schedule.

\section{Task 4.6 - Facility Disposition}

Vortec has been responsible for maintaining existing equipment purchased under Phase 3 of this project as well as DOE owned equipment at the pilot plant facilities located at the University of Pittsburgh Applied Research Center (U-PARC). The following property management reports have been provided to DOE under this contract:

1. Report of Contractor's Property Management System

2. Annual Report of Property in the Custody of Contractor

3. Report of Physical Inventory of Capital Equipment

4. Report of Termination or Completion Inventory.

\section{Task 4.7 - Subsystems Construction}

Construction of the Subsystems is to be initiated upon authorization by DOE. Authorization to proceed with construction has not yet been received. Therefore, construction activities have not been initiated.

\subsection{PROCESS DESCRIPTIONS}

This section is divided into two major subsections. Section 4.3.1 provides a process description of an Integrated Waste Pretreatment \& Vitrification Plant as developed under Phase 3 of the project. Section 4.3.2 describes a Limited Demonstration Facility as developed under Phases 3A and 4 of the project.

\subsubsection{Process Description - Integrated Pretreatment \& Vitrification Plant}

An integrated system design for a waste pretreatment and vitrification plant was developed under Phase 3 of the project. The major process subsystems of the integrated plant design include:

- Feed Preparation and Blending Subsystem

- Cyclone Melting System (CMS®)

- Glass Product Handling Subsystem

- Air Pollution Control Subsystem

- Waste Water Treatment Subsystem

The relationship of these subsystems is shown in Figure 4.3.1-1, and an artist rendering of the integrated pretreatment/vitrification plant is shown in Figure 4.3.1-2. 


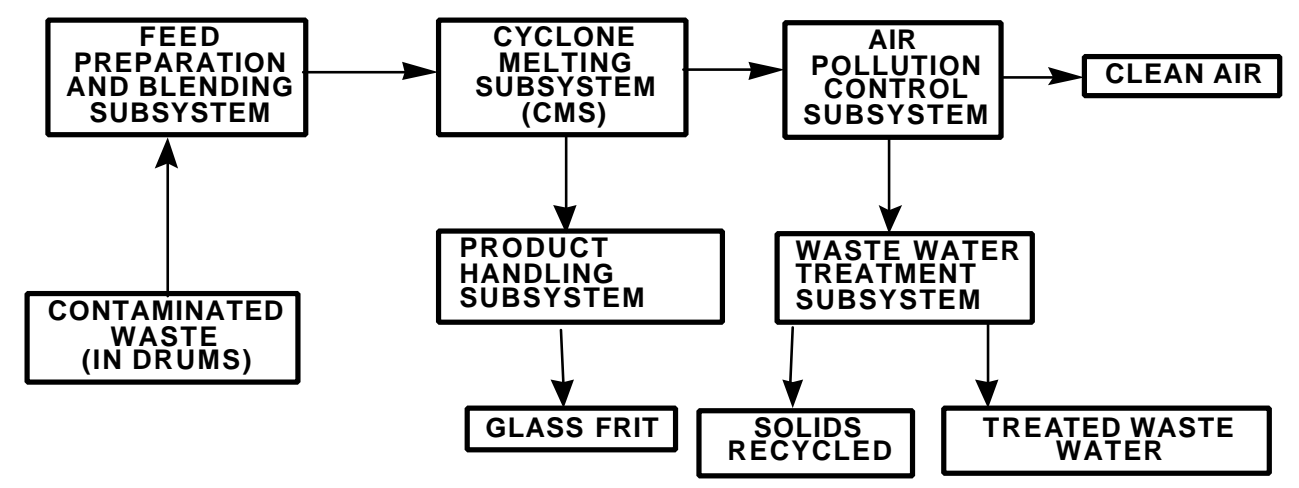

Figure 4.2.1.2-1 Block Diagram of Integrated Waste Pretreatment/Vitrification Plant 


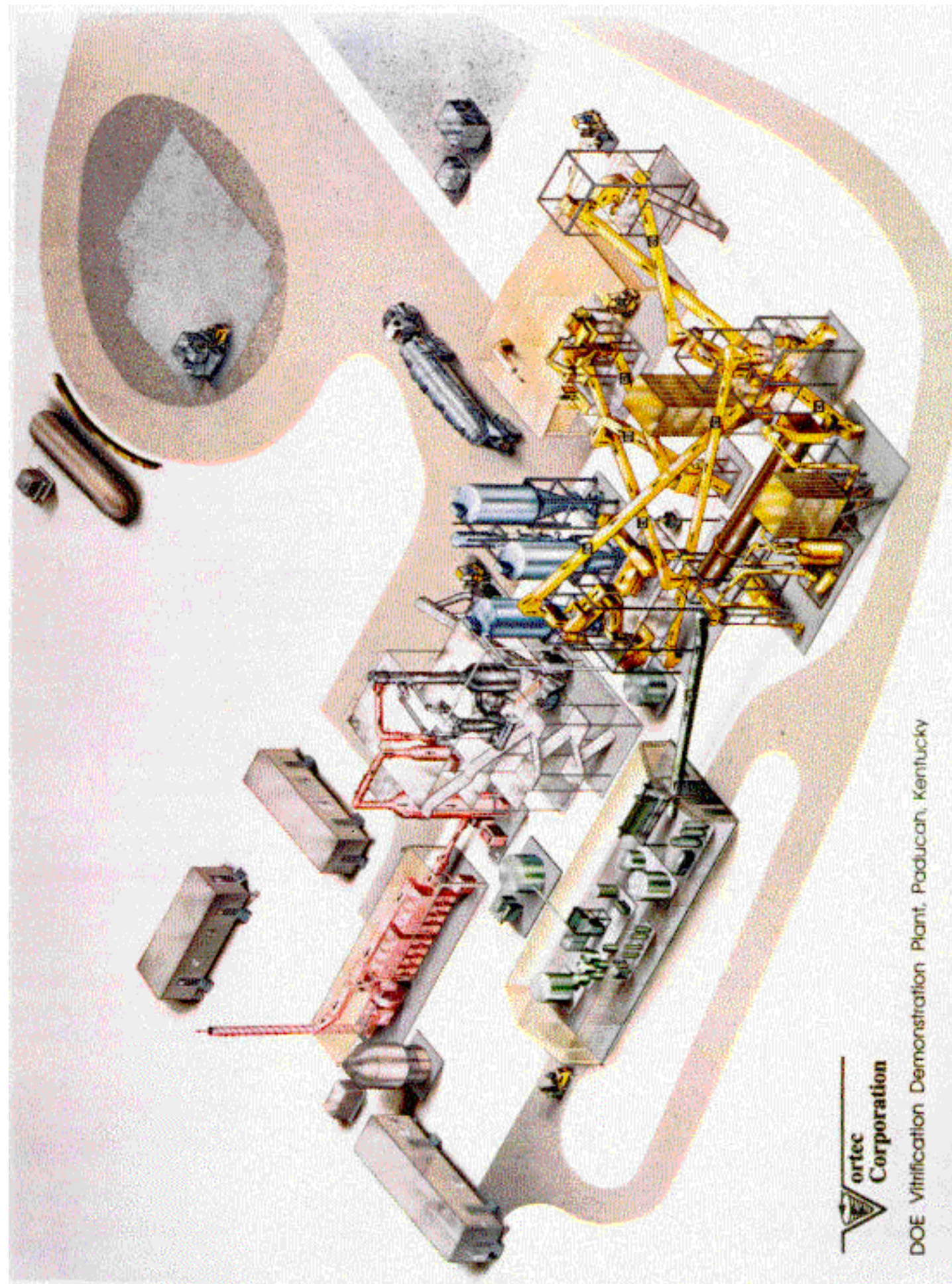

Figure 4.2.1.2-2 Artist Rendering of Integrated Waste Pretreatment/Vitrification Plant 
The combination of the feed preparation and vitrification allows a wider spectrum of waste types to be processed. The feed preparation system also provided a more homogeneous product to the vitrification plant, thus resulting in better quality control on the glass product produced. It also improves operational reliability and safety of the vitrification system while reducing the maintenance requirements for the vitrification system. Descriptions of the process assemblies that make up the integrated plant design are presented below.

\subsubsection{Feed Preparation Subsystem}

The feed preparation subsystem accepts drummed waste materials and transforms them into a dry bulk powder that meets the size and moisture specifications required by the Vitrification system. The feed preparation requirements include: shredding, drying, crushing, metal separation, plastic separation, grinding, and screening. The subsystems and assemblies that provide these functions are described below.

The process begins with the soil being delivered to the demonstration plant in sealed metal drums. The drummed, contaminated soil is stockpiled outside, near the northeast entrance to the facility. The drums are processed through a primary and secondary shredder, which reduces any rock or large materials in the drums to nominally 1 to 3 inches in size, and the drum metal and drum liner to strips 2" wide by varying lengths. The pair of shredders in series serves to minimize the number of very long strips of metal resulting from shredding the drum. This size reduction is used to prepare the waste materials and the drum for input to an indirect heated homogenizer/homogenizer/dryer. A hood over the shredder station is connected to a dust collector and induced draft fan, which provides a negative pressure and allows outside air infiltration into the shredding assemblies. This flow of outside air infiltration will prevent the escape of any dust generated during the drum shredding operation.A belt conveyor transports the shredded material to the indirect heated homogenizer/homogenizer/dryer. The soil is assumed to contain up to $30 \%$ water by weight, and a rotary homogenizer/homogenizer/dryer has been proven to dry the soil to a free flowing condition. The dry free flowing soil condition is required both for grinding and for proper operation of the pneumatic batch handling system located down stream of the homogenizer/homogenizer/dryer. Indirectly heated, closed-circuit air is used as the homogenizer/dryer medium in a rotary homogenizer/dryer. The moisture absorbed in the air drying media is passed through a condenser, which reduces the temperature to $125^{\circ} \mathrm{F}$ (dew point at these conditions is $189^{\circ}$ ) to condense out the moisture removed from the waste material. The captive drying media (air) is then released to a heat exchanger and re-circulated back through the homogenizer/dryer. The condenser has its own cooling tower, pump, and heat exchanger assemblies. The liquid removed by the condenser assembly is pumped to the plant's wastewater treatment system.

The rotary homogenizer/dryer operates on propane and is provided with its own air and fuel controls. The drying air is heated indirectly, as required to maintain the exhaust temperature from the heater to a maximum of $325^{\circ} \mathrm{F}$. The flue gas, which is not contaminated, is exhausted through a stack. The drying medium (air) is passed through a high efficiency pulse-jet bag house dust collector to capture any fine particulates remaining in the drying circuit. 
The homogenizer/dryer is designed to minimize any air infiltration into the drying circuit. The homogenizer/dryer feeding assembly and the discharge breachings are equipped with low-leakage seals. Double gate air locks provide the means for discharging the dried feed materials. The fine materials collected in the drying circuit pass through a rotary air lock to the mechanical conveying subsystem. Up to $500 \mathrm{lb}$./hr. of infiltrated air will be vented by a small fan and directed to the Vitrification process. This maintains a negative pressure in the drying circuit.

Material leaving the homogenizer/dryer is discharged onto a belt conveyor and subsequently enters the crusher. The crusher reduces the waste material stream to a minus two inch (2") product and is discharged to the separator feed conveyor.

A rotating drum magnet at the front end of the separator feed conveyor is used to remove the drum pieces and other ferrous metal strips. These metal scraps are deposited in a ST-90 box container for removal to a storage area on the site.

The material stream then enters a scalping screen, where minus 2 inch material is separated and deposited on the 1/4" screen conveyor. The plus 2 inch material is passed through an air knife for plastic separation. The segregated plastic stream is discharged into ST-90 containers for storage. A cyclone dust collector and bag-house collect any fines carried in from the separation air stream. The air used in this system is recycled.

The material stream then enters a vibrating screen where minus $1 / 4$ " material is separated and deposited on the mill feed conveyor. The minus 2 " plus the $1 / 4$ " material is fed to the rotary air separator for plastic removal. The segregated plastic stream is discharged into ST-90 containers for storage. A cyclone dust collector and bag-house collects any fines carried in the separation air stream. The air used in this system is recycled.

The waste material remaining in the stream is discharged to the mill feed conveyor. An electronic metal detector is installed on this conveyor. A pneumatically operated diverter gate at the discharge of the mill feed conveyor will automatically direct unwanted nonferrous metal to an ST-90 container located at grade.

The normal material flow from the mill feed conveyor enters a 30 mesh screen to separate out the minus 30 mesh (595 microns) material which then passes to the sized soil vacuum conveying system. The plus 30 mesh material from the screen is discharged into a grinding mill, which reduces the particle size. Material from the mill is returned to the 30 mesh screen. Air and fines from the mill pass through the mill dust collector. The collected fines are sent through a rotary air lock to the sized soil vacuum conveying system. The minus 30 mesh product from the grinding mill is also discharged to the sized soil vacuum conveying system.

The sized soil vacuum conveying system receives material from three points: the grinding mill; the mill dust collector, and the 30 mesh screen. The dried and sized soil is conveyed to the top of the soil silo. The vacuum system is closed loop with a heat exchanger to reject excess heat and an in-line filter upstream of the blower. 
Nuisance dust collection points are provided to contain fugitive dust emissions during drum shredding and throughout the feed preparation system. These materials are discharged to and collected in the mill dust collector.

Because the grinding subsystem cannot accommodate plastic materials, these materials are separated prior to the grinding operation.

The shredders, homogenizer/dryer, crusher, vibratory screens, mill, and the belt conveyors are totally enclosed. The gases leaving the mill dust collector pass on to HEPA filters. The HEPA filters are arranged in parallel so one set of filters can be serviced while the other set is on line. The differential pressure causes an alarm to notify the operator that the filters have to be changed. The clean gases leaving the HEPA filters are released to the atmosphere through the feed preparation blower.

\subsubsection{Batch and Blend Assembly}

The major components of the feedstock blending and storage assembly are: soil storage silo, two glass additive storage silos (limestone and soda ash), a weigh hopper, weighed material transporter, a feedstock blending tank, dual blended batch transporters, a rotary air lock, and an air compressor. The system is controlled by the main PLC. The weigh hopper receives material from both the soil and glass additive silos. The weighed material is conveyed to the batch blending tank via the weighed material transporter. The batch is air blended in the blend tank. Dual transporters with inlet isolation valves and an outlet rotary air lock connecting the second transporter tank to the feed line, permit the blended batch to be pneumatically fed to the Vitrification system.

Batch feed rate is established by means of logging the weight and time of each batch mix into the weigh hopper. During test operations, the size of a given batch can be optimized to provide sufficiently frequent data to accurately indicate batch feed to the CMS®.

\subsubsection{Vitrification System}

The process units of the melting (vitrification) system (CMS®) include: air delivery, fuel delivery, cyclone melting system (CMS $®)$, and a recuperator.

The CMS $®$ consists of three major assemblies: a counter-rotating vortex (CRV) reactor, a cyclone reactor, and a separator reservoir.

The reaction air for the process comes from the air blower. Air enters the Recuperator where it is preheated by exchange with off-gases to approximately $1200^{\circ} \mathrm{F}$. Preheated air from the Recuperator enters the CRV reactor inlet arms.

Propane can be delivered to either the CRV reactor inlet arms or the lid. The typical flame safety controls are provided (e.g. automatic shutdown on loss of flame, low gas pressure limit, double block and bleed valves, and a high gas pressure limit). A flow control valve sets the mass flow of propane to 
the CRV reactor. The air flow controller and the propane flow controller are interconnected by a ratio controller to maintain the air to fuel ratio and also control the air lead/lag when changing firing rates.

During start-up, the temperature of the unit will be increased at a rate of $50^{\circ} \mathrm{F} / \mathrm{hr}$. in order to prevent thermal shock to the refractory. Main gas to the CRV reactor cannot be turned on until the CRV reactor has reached $1400^{\circ} \mathrm{F}$, and at this temperature propane will auto ignite.

Like the CRV reactor, the cyclone melter is a refractory lined, water cooled, carbon steel vessel. The high inlet velocity causes a cyclonic flow to occur within the melter. The centrifugal forces caused by the gas dynamics throw the molten material to the walls where glass forming reactions initiated in the CRV reactor are completed. The molten glass and the hot gases then pass on to the separator reservoir,

Between the cyclone melter and the separator reservoir are two thermocouples, which measure the glass temperature as it leaves the cyclone melter. These thermocouples are used during melting operations to control the firing rate of the process and maintain a constant glass temperature. Should the glass temperature start to fall, the firing rate will be increased; likewise, should the temperature increase, the firing rate will drop.

The separator reservoir is a refractory lined and insulated carbon steel vessel. Inside the separator reservoir the gas velocities drop dramatically allowing a final separation of glass and gas, Thermocouples installed in the separator reservoir provide the operator with a temperature profile during operation. A pressure transmitter monitors the pressure within the separator reservoir, which is used as the balance point for the balanced draft operation. Should the separator reservoir pressure rise above setpoint, the ID fan speed will be increased to reduce the pressure, Should the pressure decrease below the setpoint, the ID fan speed will be decreased to increase the pressure.

The molten glass leaves the separator reservoir and enters a glass channel. The glass channel has propane fired burners to maintain glass temperature during operation and to bring the glass channel up to operating temperature during heat up. Molten glass leaves the glass channel by flowing over a weir and falling into the glass handling system. Hot gases exit the separator reservoir and flow upward through the Recuperator where the waste heat is used to preheat the air. Flue gases leaving the Recuperator pass on to the air pollution control system.

\subsubsection{Glass Product Handling Subsystem}

The glass product handling subsystem consists of a water quench tank and a drag conveyor. Hot glass from the separator reservoir falls into the glass quench tank. The quench tank rapidly cools the glass to a temperature below $200^{\circ} \mathrm{F}$. The drag conveyor pulls the glass from the quench tank and allows it to de-water as it goes up the conveyor incline. The speed of the conveyor will be adjusted with a variable speed drive to allow residual heat in the cullet to evaporate as much water as possible from the cullet prior to being discharged into ST-90 containers. 
Two ST-90 containers can be placed under the drag conveyor at the discharge end in parallel. Motorized valves will sequence the filling of the boxes alternately. The full ST-90 containers will be removed and placed in the treated container storage area for pick-up and disposal by DOE-Paducah.

Hot water from the quench tank will be circulated through a heat exchanger to be cooled by water from the cooling tower. Water from the quench tank will flow over a weir into a surge tank, which has a 5 minute residence time. The weir is provided to keep particulate out of the circulating loop. The cooled water is re-admitted to the quench tank as a spray to minimize the potential for dust generation.

\subsubsection{Pollution Control Subsystem}

The air pollution control subsystem has been designed for a maximum uncontrolled particulate carryover rate of $5 \%$ of the batch feed rate. The off gas (flue gas) from the recuperator flows into the venturi scrubber. For a maximum efficiency from the scrubber at varying flow rates, a variable throat venturi was selected.

The off gas from the venturi scrubber passes on to a separator where particulate laden water is separated from the off gas. Water is collected in a sump at the base of the separator and is recycled back to the venturi scrubber by the recycle pump.

The $\mathrm{pH}$ of the recirculating water will drop as acid gases are absorbed from the flue gas. Caustic is added to the recycle line as required to maintain the $\mathrm{pH}$ set point. Water is blown down to the wastewater tank as required.

The off gas leaves the separator and passes on to the wet electrostatic precipitator (WESP) irrigation chamber. A WESP is very efficient in collecting water droplets; if particulate passing through the WESP is uniformly coated with water, then the particle collection efficiency will be high. The irrigation chamber contains a fogging spray to assure that the particulate is coated with water prior to entering the WESP. Any excess water with particulate flows out of the irrigation chamber and into the WESP blowdown tank.

The off gas leaves the irrigation chamber and enters the WESP (ME-501) through the top of the unit. As the water droplets with the entrained particulate pass through the electrostatic field, they will be attracted to and collected by the collector plates. As the water rolls down the collector plates, it will effectively clean the plates. If additional cleaning is required, spray nozzles located above the collectors can be turned on to wash the plates with clean water. Water and particulate will pass out the bottom of the WESP into the blowdown tank while the off gas passes out the side and on to the process off gas heater. The blowdown pump is cycled on and off by level control.

The process off gas heater receives the off gas and raises the temperature to $250^{\circ} \mathrm{F}$ to elevate the dew point prior to entering the high efficiency particulate air (HEPA) filters. At this temperature, any water left in the off gas will be in the vapor phase and the gas dew point will be sufficiently high to prevent moisture from condensing in the HEPA filters 
HEPA filters are provided for final particulate clean-up. The filters are in parallel so one set of filters can be active while the other set is being replaced. Differential pressure across the filters is monitored to indicate when the filters should be changed out.

The gases are discharged from the Process Blower through the Process Exhaust Stack where it is vented to the atmosphere. The stack is equipped with a continuous emissions monitoring system, which measures opacity, $\mathrm{HCl}, \mathrm{CO}_{2}, \mathrm{CO}, \mathrm{O}_{2}, \mathrm{SO}_{2}$, and $\mathrm{NO}_{\mathrm{x}}$. The analyzer signals are sent to the PLC for data recording, and calculations.

\subsubsection{Waste Water Treatment Subsystem}

Wastewater streams from the venturi scrubber, the WESP, the vapor recovery condenser pump, the filter press filtrate pump, and any recycle stream from the treated effluent pumps flow into the wastewater flocculation tank. A sodium hydroxide solution (under $\mathrm{pH}$ control) and a polymer mixture (under flow control) are added to the wastewater flocculation tank. The sodium hydroxide solution is added to raise the $\mathrm{pH}$ of the liquid to a set point of 9.2 to form insoluble metal hydroxides, while polymer is added to aid in flocculating the small metal hydroxide particles into settleable solids.

The contents of the wastewater flocculation tank flow by gravity into the wastewater holding tank. The wastewater in the wastewater holding tank overflows from near the top of the tank into a clearwell, from which it is pumped to the following steps.

Wastewater is pumped first through the wastewater heat exchanger $(\mathrm{HX}-600)$ to reduce its temperature to $100^{\circ} \mathrm{F}$. The cooled wastewater is then introduced to reaction tank A. Sulfuric acid and sodium metabisulfite solutions are added to this tank to keep the $\mathrm{pH}$ of the wastewater at 3.0 and to reduce any hexavalent chromium to trivalent chromium. The reaction tank is constantly agitated and the $\mathrm{pH}$ and oxidation-reduction potential are measured. The $\mathrm{pH}$ measurement is used to control the addition of sulfuric acid, and the ORP measurement is used to control the addition of sodium metabisulfite.

The wastewater then flows over a weir to reaction tank B. Sodium hydroxide solution is introduced to raise the $\mathrm{pH}$ of the wastewater to 7.5 to 8.0 to precipitate chromium hydroxide.

The contents of reaction tank B flow over a weir to the flocculation tank. Diluted polymer mixture is added to flocculate and settle solids in the clarifier. Sodium sulfide can also be added to precipitate any remaining dissolved metals as insoluble metal sulfides.

The wastewater overflows a weir into the clarifier. Solids settle to the bottom of the clarifier and are pumped continuously to the sludge holding tank skid. The clarified wastewater overflows into the clarifier clearwell, which acts as a holding or surge tank for the clarified wastewater before it is pumped to the polishing filter skid.

The chemically treated wastewater is then filtered to remove any remaining particulate material that is suspended in the clarified wastewater and to keep such material from plugging or fouling the operation of the ion exchange resin. 
The filtering skid is composed of three equivalent multimedia filters. The polishing filter skid can operate in two modes. The first mode, the filtering mode, is when all three filters are operating in parallel, and all of the filtered wastewater is input to the ion exchange skid. The second mode, the backwash mode, is when one filter is backwashed and the other two filters, operating in parallel, are feeding the filter in backwash. All three filters can be backwashed sequentially. When the polishing filter skid is in backwash mode, the backwash is input to the filtrate tank skid. The differential pressure between the inlet and outlet manifolds for the three filters is measured to control the initiation of the backwash mode.

The filtered wastewater is treated though ion exchange resin to remove specific radionuclides and heavy metals that are still in solution within the wastewater effluent.

A bank of three ion exchange cells accomplishes this purpose. The piping of the skid allows for the wastewater to flow through the cells in series or parallel. Treated water is pumped to the effluent tank skid, where it is held for analysis prior to discharge to DOE's Outfall No. 1.

Sludge generated from the wastewater holding tank and the clarifier is pumped to the sludge holding tank. From this tank, the sludge is pumped to the plate and frame type filter press for dewatering.

During sludge dewatering, the filtrate from the filter press is sent to a filtrate tank When the filter press is opened, the dewatered filter cake is dropped into a live bottom hopper. The sludge screw conveyor conveys filter cake out of the hopper and transfers it to the inclined screw conveyor C8. The C8 conveyor transfers the filter cake either to the rotary homogenizer/dryer for recycle or to ST-90 boxes for disposal.

The filtrate from this process is returned to the wastewater flocculation tank for retreatment through the system.

The treated wastewater is sent to the effluent tank skid, where it can be recycled as make-up water to the venturi scrubber, the glass quench storage tank, and the wet ESP or discharged.

Treated effluent that is not recycled in the wastewater treatment system is sent to one of the three effluent holding tanks. The effluent holding tanks each hold the treated effluent from one shift until an analysis is performed. After completing the analysis, the treated effluent is either sent to the wastewater outfall, discharged into DOE tank trucks, or passed through the polishing filter and ion exchange skids.

Potentially contaminated rainwater is collected and treated through the wastewater treatment system.

\subsubsection{Process Monitoring and Control Subsystem}

The process monitoring and control subsystem consists of the sensors, electronics, instrumentation, operator's stations, computers, and programmable logic controllers (PLC) to control the Demonstration Plant in real time, gather data for analysis on system and equipment performance, and monitor process off gas. The human interface is included as well as hard copy information gathered by hand.

The system is fault tolerant and is capable of being shut down in emergencies in a controlled manner using structured logic. Interlocks control a wide range of failures. Central alarms in the control trailer detect off nominal conditions and emergency alarms are sounded in the plant area. An uninterruptible 
power supply assures computer power availability during power outages and is independent of the emergency power generator. The system has been designed so that automated valves fail in a safe position.

Proven industrial controls and electronics are used. All indoor equipment is NEMA 12 and all outdoor equipment is NEMA 4 or 3R. Redundant programmable logic controllers enhance reliability. Multiple monitors are capable of being switched to allow individual subsystem processes to be monitored in the control trailer.

A data acquisition subsystem (data logger) assures that key data for analysis of system and equipment performance is obtained. The data acquisition function resides within the personal computers to assure no loss of continuity in data. Selected data will be collected automatically after loss of power for 60 minutes. In addition to collecting and recording data for process and equipment evaluation, and trend analysis, the system incorporates a continuous emissions monitoring system for process emissions surveillance. Record Sampling is used to document meeting emission standards.

The system consists of the following:

- A main Operators Console with two operator stations and a printer, two historian file servers, two log printers, two modems, and coax links

- $\quad$ A main Control Panel, MCP-1 with necessary input/output and two redundant programmable logic controllers

- $\quad$ An uninterruptible power supply system

- Controlling software

- $\quad$ Instrumentation as defined in the P\&ID and specifications

The operator's console consists of two fully independent desk top operator stations located in the control trailer. The operator's console houses the equipment and provides work space for the operators. Since no components are shared, failure of one station will not affect operation of the other.

Each station has the necessary software to configure all system devices. A critical alarm display unit monitor provides split screen operation so that one section is devoted to a time ordered, alarm review scroll and the other sections dedicated to the alarm status of 8 critical process alarms.

The system will provide the following displays:

- Summary Display - overview of multiple groups in a single display

- Indicator/Controller Face Plates - 8 or more points on a single display in the face plate format

- Point summary display - Analog input and output signal values and motor command and status values

- $\quad$ Process Graphic Displays - up to 100 custom graphic displays based on the P\&ID

- $\quad$ Historical Trends - up to 250 historical trend points

- Diagnostic Displays - Network diagnostics with on screen help for troubleshooting network failures 
- Alarm handling - all alarms with archive capability

Control is exercised by a set of four PLCs. The control system is automated to the maximum possible extent. Process control valves are operated remotely from the operator's console in the control trailer. Controllers have the capability to be manually operated so that the combustion air blower and cooling water pumps can be operated in case of system failure. Loop controllers can be operated in a manual mode for control of valves, variable frequency drives and other selected equipment.

Communications among the system components is accomplished over redundant, high speed data highways capable of providing 2 second updates for all highway functions including displays, controller changes, and alarm reporting.

\subsubsection{Utilities Subsystem}

\section{$\underline{\text { Power Distribution System }}$}

Primary electrical power is supplied by DOE-Paducah through a bank of transformers to the Vortec system. Emergency power is supplied from a $400 \mathrm{kva}$ diesel emergency generator through a $400 \mathrm{amp}$ automatic transfer switch. The emergency generator supplies back-up power for: combustion air blower, continuous emissions monitor, cooling water supply pumps, cooling tower fan, instrument air compressor, and emergency lighting.

Skids are independently provided with power through a skid mounted disconnect. Each skid is prewired and checked out before shipment to the site. Instrumentation is installed on site to prevent damage during shipment.

\section{Cooling Water}

The cooling water system provides cooling water to remove excess heat from the cyclone melting system (CMS $®)$, wastewater cooling system, and the glass quench cooling system. The cooling tower is an induced draft, counter flow, closed loop, evaporative cooler with three (3) $10 \mathrm{hp}$, TEFC, direct drive propeller fans, complete with electric water level control, temperature control, electric basin heater, galvanized steel coil, pressurized water distribution, and PVC mist eliminators. The cooling water rack provides a single location for operator monitoring and control of the cooling water supply and return to the CMS ${ }^{\circledR}$. The glass quench cooling system serves to remove heat from the glass quench water, which overflows from the glass drag conveyor, and to pump the cooled quench water back to the conveyor spray headers. The wastewater cooling system serves to reduce the wastewater temperature to enable the use of plastic materials.

The cooling tower is designed for a flow rate of $568 \mathrm{gpm}(40 \%$ propylene glycol \& $60 \%$ water) with a cooling water return temperature of $110^{\circ} \mathrm{F}$. The total capacity of the system without the reservoir tank 
is 1200 gallons. The reservoir tank has a capacity of 250 gallons. The required cooling water supply temperature is $93^{\circ} \mathrm{F}$, total heat duty of 4,540,000 BTU/hr, design ambient wet bulb temperature of $78^{\circ} \mathrm{F}$, and cooling water design pressure $100 \mathrm{psig}$. The cooling tower will remove heat from the cooling water circulating in the coil by recirculating water over the outside of the coil and blowing air up through the wetted coil. The water that is recirculated over the outside of the coil is stored in the pan section at the bottom of the unit, and is pumped over the coil through spray nozzles by the spray pump mounted on the end of the unit.

The make up water is supplied from the utility water system. An electric immersion heater is turned on automatically to prevent freezing of the reservoir when the tower is shut down. The thermostat is to be set at $42^{\circ} \mathrm{F}$.

Two cooling water pumps are provided for cooling water recirculation. Only one will operate at a time, the other acts as a $100 \%$ spare.

The cooling water will enter the glass quench heat exchanger at a maximum rate of $280 \mathrm{gpm}$ and a temperature of $93^{\circ} \mathrm{F}$ before returning to the cooling tower. Heated water leaving the glass quench tank at $135^{\circ} \mathrm{F}$ will overflow to the glass quench surge tank.

\section{$\underline{\text { Fuel Supply }}$}

Propane, which is used as fuel for the rotary homogenizer/dryer, CMS®, and the off-gas air heater, is trucked to the site and stored in the plant propane storage tank. The propane system consists of a liquid propane tank propane pump and a vaporizer all supplied with the necessary instruments, valves, and safety controls.

\section{Plant Compressed Air}

A plant compressed air system (ME-700) consisting of an inlet filter, air compressor, aftercooler, water separator, and an air receiver is provided for plant utility air requirements. Utility air is also passed through a coalescing filter, regenerative homogenizer/dryer, and an after filter in order to provide plant air with a $-40^{\circ} \mathrm{F}$ dew point for instrumentation.

\subsubsection{Skids/Facility Foundations/Structures/Piping}

\section{$\underline{\text { Skids }}$}

Each skid or assembly has been designed to be a modular unit that can be transported over the roads. The envelope will meet Department of Transportation requirements for height, length, width and weight.

Each skid has been pre-wired and pre-piped with the interface points for connections between skids and other equipment being minimized. All skid internal wiring is routed to a central panel which in turn will connect to an I/O panel. The I/O panel will receive inputs from outside the skid and will provide the 
interface with the process monitoring \& control system. In addition, electrical control panels will be provided on each skid to receive outside power. Interconnecting piping between the skids and other process equipment is intended to be flexible hose where possible.

A cable tray/hose tray support has been routed down the center of the skid arrangement, which allows take-off to each individual skid. This tray arrangement will house both hose on the bottom layer and electrical cables on the top layer. Hose and cable leaving the tray will be laid on the ground between the tray and the connecting points on the skids.

Each skid has been designed to operate outdoors. However, since there is no insulation or heat tracing (to save cost) when ambient temperature reaches freezing, each skid will be drained and compressed air blown through the piping to remove any water which potentially could cause freezing problems.

\section{$\underline{\text { Structures }}$}

The structural design criteria was tailored after the following standards or codes:

- DOE 6430.1a (1989): United States Department of Energy General Design Criteria

- UCRL-15910 (1990): Design and Evaluation Guidelines for Department of Energy

- $\quad$ SDC 4.1 (1993) :

Facilities Subjected to Natural Phenomena Hazards

- ASCE 7-93 (1993):

Hanford Plant Standards : Arch-Civil Design Criteria.

- 1988 UBC:

Minimum Design Loads For Buildings and Other Structures

Uniform Building Code, 1988 Edition

The facility was classified as a Non-nuclear, General Use, Safety Class 4, in Seismic Zone 2B. The Non-nuclear classification was based on guidance in DOE 1027-92. In accordance with these standards, the structural design gravity loads and wind loads were established from ASCE 7-93 with the following assumptions:

- Gravity loads: $\quad$ CMS $\quad$ Structure Elev. Platform 125 psf Filter Press Platform 100 psf

$\mathrm{CMS}{ }^{\circledR}$ Structure grade slab $\quad 250 \mathrm{psf}$

- Wind loads based on: $\quad$ Basic Wind Speed $70 \mathrm{mph}$

Exposure C

The Seismic design loads were established from 1988 UBC in conjunction with SDC 4.1 and UCRL 15910 assuming the following parameters:

$\begin{array}{lll}\text { - } & \text { Zone } & 2 \mathrm{~B} \\ \text { - } & \text { Importance Factor } & 1 \\ \text { - } & \text { Dampening Factor } & 5 \% \\ \text { - } & \text { CMS® Structure seismic factor } & 0.0412\end{array}$


The CMS ${ }^{\circledR}$ structure was designed as an unenclosed, steel braced, frame tower with a ground floor and 3 elevated floors braced with horizontal truss systems. The filter press support platform is an $\mathrm{x}-$ braced, steel structure with a horizontal truss braced floor system. Both structures were designed as field bolted, portable structures. To simplify and facilitate portability, the bracing in the CMS ${ }^{\circ}$ structure was designed as single member compression/tension diagonal braces. The CMS® structure was analyzed using the STAAD-III structural analysis software and checked for compliance to the 1989 ASD structural steel specification. The foundation was analyzed as a mat foundation assuming an allowable soil bearing pressure of 3000 psf. Concrete design was done in accordance to the 1983 ACI 318-89 ultimate strength design method.

\subsubsection{Limited Demonstration Plant Process Description}

The process for the limited plant demonstration consist of primary process elements of the pretreatment system described above and is herein referred to as the Material Handling and Conditioning System. The basic process design for the limited demonstration plant was developed under Phase $3 \mathrm{~A}$ of the project and refined under Phase 4.

\subsubsection{Description of Material Handling and Conditioning System}

The Material Handling and Conditioning System consists of a portion of the feed preparation system for the full-scale integrated pretreatment/vitrification plant; for this reason, this phase of work is referred to as the "Limited Demonstration. The system to be installed and demonstrated under this scope of work is as shown on the Process Flow Diagram in Figure 4.3.2.1-1. The Material Handling and Conditioning facility will consist of a drum weighing and loading assembly, shredding assembly, homogenization/drying process, ferrous metals separation assembly, prepared waste delivery/loading assembly, a comprehensive fugitive dust control system, the propane fuel subsystem, the compressed air system, and PLC-based controls and power distribution. The limited demonstration plant is located outside the security fence at Paducah and located on approximately five acres of land provided by the site. Approximately a third of the land set aside for the vitrification plant will be used for Material Handling and Conditioning Subsystem.

The Material Handling and Conditioning Subsystem begins with clean soils drummed in sealed metal barrels, prepared by BJC and delivered to the Vortec facility for the limited demonstration project. The drummed materials will be prepared in accordance with recipes defined in the Test Plan. The sealed drummed soils will be weighted and loaded into the Primary Shredder via the Manipulating Arm and Skip Hoist. Materials will pass from the Primary Shredder to the Secondary Shredder to complete the drum shredding process. Shredding will yield metal strips no larger than 2" by 6" and break down rocks, concrete and other objects for good metal separation and flow characteristics.

Upon leaving the secondary shredder, the metal strips and drummed contents are delivered to a belt conveyor transporting and elevating the shredded materials to the Rotary Drum Homogenizer/homogenizer/dryer where moisture content will be reduced by contact with heated air. 
Dried materials discharge from the Rotary Drum Homogenizer/Homogenizer/dryer onto a belt conveyor, which transports them to the conditioned soil loading subsystem. At the head of the conveyor, a tramp iron magnet removes ferrous materials from the material stream. Separated metals are - discharged to an ST-90 box for DOE disposal. The conditioned soil will discharge from the belt conveyer to the waste delivery/loading assembly into ST-90 boxes for DOE disposal.

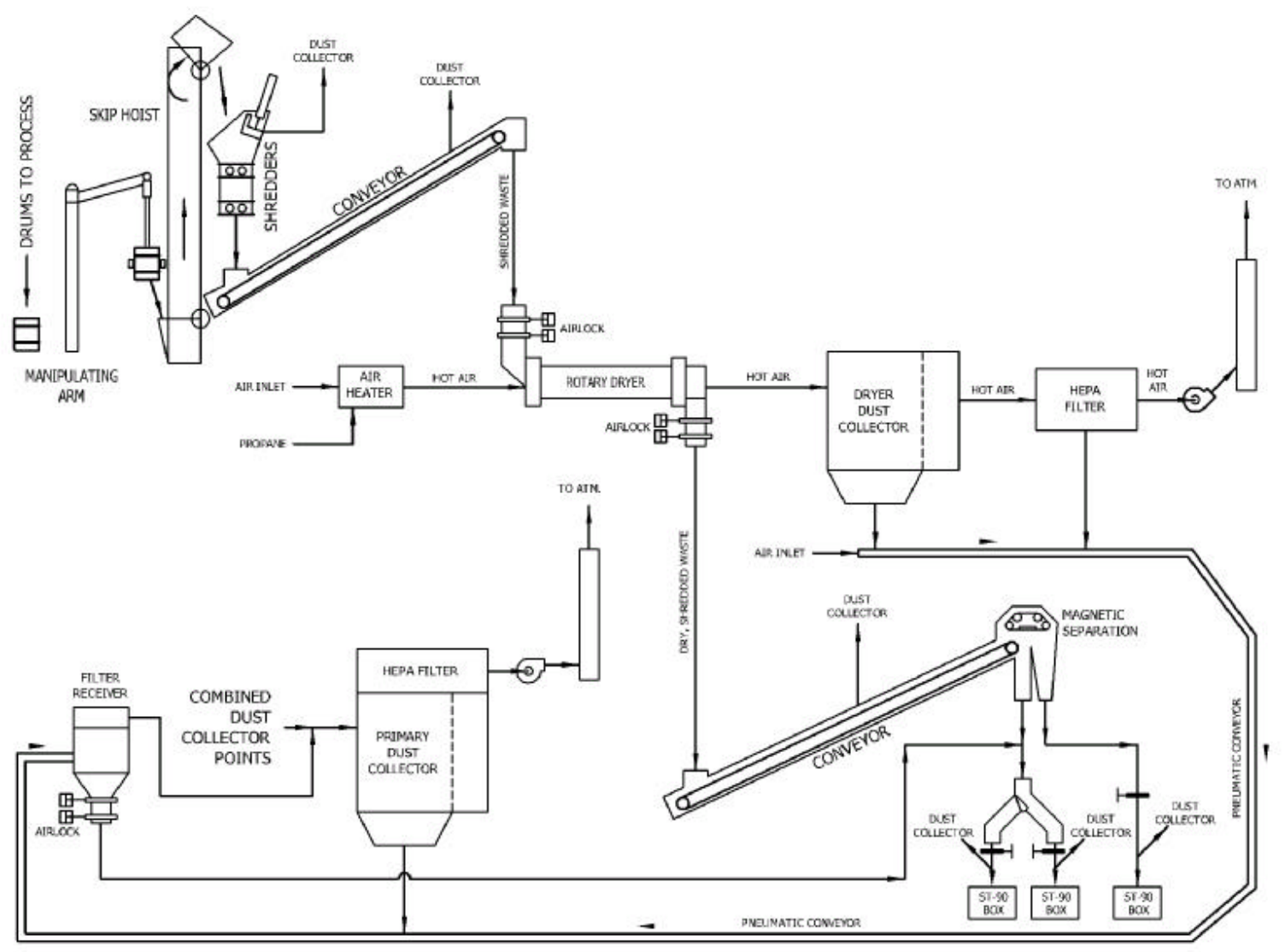

Figure 4.3.2.1-1. Process Flow Diagram

Necessary process equipment ancillary to the soil preparation and conditioning facility include a homogenizer/homogenizer/dryer air heater system, a treatment subsystem for the homogenizer/homogenizer/dryer exhaust air stream, dust control subsystem, the propane subsystem, the compressed air subsystem, power and controls.

The soil is assumed to contain up to $30 \%$ water by weight, and a rotary homogenizer/homogenizer/dryer has been provided to dry soil to a free flowing condition. Drying air exhausted from the homogenizer/homogenizer/dryer is passed through a bag house and HEPA filters for control of particulate prior to discharge to the atmosphere. 
The rotary homogenizer/homogenizer/dryer air heater operates on propane and is provided with its own air and fuel controls. The drying air is heated as required to maintain the exhaust temperature from the heater to maximum of $600 \mathrm{~F}$.

The homogenizer/homogenizer/dryer operates under a slight negative pressure and is designed to minimize any air infiltration into the drying circuit. The homogenizer/homogenizer/dryer feeding assembly and the discharge breaching are equipped with low-leakage seals. Double gate airlocks provide the means for charging and discharging materials to and from the Rotary Homogenizer/homogenizer/dryer.

All of the soil processing equipment (shredders and conveyors) has been designed to be enclosed and discharge to a dust control subsystem to minimize and possibly eliminate fugitive dust emissions. The dust control subsystem provides the capability to withdraw air from all of the soil processing equipment and filter it through a dust collector and High Efficiency Particulate Air (HEPA) Filter prior to discharge to the atmosphere. The dust control subsystem consists of the ductwork, Nuisance Dust Collector/HEPA filter, blower, and stack. The Homogenizer/homogenizer/dryer exhaust gas is similarly vented through a dust collector and HEPA filter. Both Dust Collectors (Nuisance and Homogenizer/homogenizer/dryer) and the Homogenizer/homogenizer/dryer HEPA filter are equipped with automatically controlled air pulse subsystems to clean the filer media. In each unit, the dust removed from the filter media drops to a live bottom hopper where it is discharged to a pneumatic conveying system for delivery to the prepared soil loading facility. In this manner, all potential dust sources during operation of the system are effectively controlled and fugitive losses are minimized or eliminated.

The propane subsystem consists of the Liquid Propane Tank and ancillary equipment. The propane subsystem is designed for $21 \mathrm{MMBTU} / \mathrm{hr}$ of propane, while the homogenizer/homogenizer/dryer heater requires a maximum of $7 \mathrm{MMBTU} / \mathrm{hr}$.

The compressed air subsystem provides both instrument air and utility air to the plant. It consists of a single stage rotary screw type compressor with air-cooled aftercooler, a heatless, regenerative, desiccant type air homogenizer/homogenizer/dryer and air receiver. The subsystem is designed to provide $800 \mathrm{scfm}$ of compressed air at $125 \mathrm{psig}$ with a $-40^{\circ} \mathrm{F}$ dewpoint. The compressor and homogenizer/homogenizer/dryer operate with manufacturer provided local control panels.

To provide transportability and ease installation, most equipment is skid mounted, meets Department of Transportation regulations, and uses flanged and/or hose connections. The systems are designed for operation outdoors at temperatures below freezing.

\subsubsection{Process Effluents}

\section{Air Emissions Points}

There will be two air emissions points, the stack venting from the Nuisance Dust Collection subsystem and the stack venting exhaust gases from the Homogenizer/homogenizer/dryer. Information for these 
two point sources is shown in Table 4.3.2.2-1. The level of emissions from these two sources under the limited demonstration scenario is low enough to constitute a minor source requiring no air permit. No hazardous air pollutants or radionuclides will be discharged and the emissions of criteria pollutants will be well below the minor source limits of 25 tons per year.

Table 4.3.2.2-1. Point Source Information ${ }^{1}$

\begin{tabular}{|l|c|c|}
\hline Source & Nuisance Dust Collection & $\begin{array}{c}\text { Homogenizer/homogeniz } \\
\text { er/dryer System }\end{array}$ \\
\hline Flow rate, acfm & 19,000 & 10,000 \\
\hline Pollutant rate, total pounds ${ }^{2}$ & & 2.64 \\
\hline Particulate & $1.45 \times 10^{-3}$ & 18.68 \\
\hline Carbon Monoxide & -- & 74.8 \\
\hline Oxides of Nitrogen & -- & $8.44 \times 10^{-2}$ \\
\hline Sulfur Dioxide & -- & 1.56 \\
\hline Volatile Organic Compounds & -- & 1968 and \\
\hline
\end{tabular}

Notes: 1. Sources of data for this table are Air Permit Application, Kenvirons, January 1998 and calculations by DOE. 2. The total pound emission rates were calculated based on processing 1000 drums of material through the soil preparations and conditioning subsystems as described in this proposal at a maximum rate of 2.25 tons per hour.

\section{Liquid Effluents}

No liquid effluents are expected from the system, other than uncontaminated stormwater runoff.

\section{Solids Discharge Points}

There will be two solids discharge points. Solids at each point will be collected in an ST-90 box for disposal by DOE. The two points are: the ferrous metals at the tramp iron metal separator, and the prepared and conditioned soil discharge point. 


\subsection{Demonstration TeSt Plan}

The demonstration testing to be performed is constrained to the testing of the Material Pre-Treatment and Conditioning System using non-hazardous surrogate materials. The Limited Demonstration Test will include a shakedown and commissioning time prior to commencing the test. The basis of the test plan is that the limited demonstration will continue for a 30-day period, using drummed clean soil and other materials to form a waste surrogate, prepared by the Bechtel Jacobs Corporation (BJC) under contract to DOE. It is anticipated that approximately 500-1000 drums of materials will be processed during the limited demonstration. Sampling and analytical work for the limited demonstration criteria will be limited to those for the equipment to be demonstrated. The demonstration criteria will include measures of the effectiveness of sealed drum feeding, shredding, drying, homogenization, ferrous metal separation, fugitive dust control, and process control.

The approach to demonstrating the Material Pre-Treatment and Conditioning System is to perform a series of trials. In these trials, drums containing surrogate materials representative of material that could be encountered at DOE facilities would be processed. The trials will demonstrate the versatility and effectiveness of the system on a range of materials including soils containing $15 \%$ to $30 \%$ moisture content, and combinations of soils and debris such as concrete chunks and steel reinforcement bars. The soils used in the demonstration will be representative of the soils local to the Paducah facility.

The demonstration contains five experiments, which will consist of process-related tests to confirm that each component of the system is functioning properly and is ready to accept materials. The demonstration period is planned to include 30 days of testing. During the demonstration period, the system will operate on one shift, weekdays only. The primary decision to be made from the test program is if the material pre-treatment and conditioning system can yield a more homogeneous feedstock appropriate for direct disposal or further treatment.

\subsubsection{Test Objectives}

To support the final decision of the suitability of this technology for conditioning and treating the material present at the Paducah site, the system will be operated with the test objectives summarized in Table 4.4.1-1.

The major objective is to demonstrate that using suitable surrogates of waste present on the Paducah site, the Vortec Material Preparation and Conditioning Systems can produce a homogeneous and dry feedstock appropriate for direct disposal or further treatment. Data collected will include material physical characteristics. Equipment data regarding metals removal, utilities consumption, and homogenizer operations will also be collected.

The data required during the trials includes performance and regulatory data. The performance data is required to complete the understanding of the operation of the system while the regulatory data ensures that the operation of the facility is in compliance with all applicable regulations and provides data for any future permitting activities. 
Table 4.4.1-1. Summary of Test Objectives

\begin{tabular}{|l|l|l|l|}
\hline No. & Test Objective Title & \multicolumn{1}{|c|}{ Test Objective Statement } & Objective Type \\
\hline 1 & $\begin{array}{l}\text { Homogenizer Drying } \\
\text { Performance }\end{array}$ & $\begin{array}{l}\text { Determine the homogenizer efficiency and the } \\
\text { percent water in the conditioned material as a } \\
\text { function of feed material characteristics and } \\
\text { material feed rate }\end{array}$ & Operating Data \\
\hline 2 & $\begin{array}{l}\text { Tramp Iron Magnet } \\
\text { Performance }\end{array}$ & $\begin{array}{l}\text { Determine magnetic metal removal efficiency } \\
\text { and the amount of magnetic metals remaining in } \\
\text { conditioned material. }\end{array}$ & Operating Data \\
\hline 3 & $\begin{array}{l}\text { Particle Size } \\
\text { Reduction Efficiency }\end{array}$ & $\begin{array}{l}\text { Determine the reduction in particle size that } \\
\text { occurs during shredding and drying. }\end{array}$ & Operating Data \\
\hline 4 & $\begin{array}{l}\text { Homogenization } \\
\text { Effectiveness }\end{array}$ & $\begin{array}{l}\text { Determine the amount of blending that occurs in } \\
\text { the system. }\end{array}$ & Operating Data \\
\hline 5 & Drum Size Capacity & $\begin{array}{l}\text { Verify the system capacity to process both 55- } \\
\text { gallon drums and 85-gallon overpacks. }\end{array}$ & Operating Data \\
\hline 6 & $\begin{array}{l}\text { System Maximum } \\
\text { Processing Rate }\end{array}$ & $\begin{array}{l}\text { Determine the maximum system processing rate } \\
\text { and the rate limiting subsystem. }\end{array}$ & Operating Data \\
\hline 7 & Utilities Consumption & $\begin{array}{l}\text { Determine the electricity and fuel required for } \\
\text { processing material on a per unit basis. }\end{array}$ & Operating Data \\
\hline 8 & $\begin{array}{l}\text { Efficiency of Dust } \\
\text { Emissions Control } \\
\text { System }\end{array}$ & $\begin{array}{l}\text { Determine the particulate emissions from the } \\
\text { nuisance dust collection vent and the } \\
\text { homogenizer exhaust vent. }\end{array}$ & Regulatory \\
\hline 9 & $\begin{array}{l}\text { Fugitive Dust } \\
\text { Emission }\end{array}$ & $\begin{array}{l}\text { Identify potential of areas which could lead to } \\
\text { fugitive dust emission }\end{array}$ & Regulatory \\
\hline
\end{tabular}

\subsubsection{Performance Data}

The demonstration test criteria will include measures of the efficiency of drying, metals separation, and particulate removal in the dust control system; the effectiveness of homogenization, fugitive dust emission control, and product handling system; the effectiveness of the back-up electrical generation system to maintain the system at a slight negative pressure in response to a loss in the primary electrical power source; and the maximum continuous processing rate. The performance data to be collected during the trials and a brief description of the methods used to collect these data are given in Table 4.4.2-1. 
Table 4.4.2-1 Performance Data for Demonstration Trials

\begin{tabular}{|l|l|}
\hline \multicolumn{1}{|c|}{ Data Required } & \multicolumn{1}{c|}{ Collection Method } \\
\hline Moisture content of feed & $\begin{array}{l}\text {-Measure amount of water added to surrogate materials during } \\
\text { preparation. } \\
\text {-Loss on drying analysis performed on grab samples from drums. }\end{array}$ \\
\hline $\begin{array}{l}\text { Moisture content of conditioned } \\
\text { soil }\end{array}$ & $\begin{array}{l}\text { Loss on drying analysis performed on grab samples of material to } \\
\text { ST-90 boxes. }\end{array}$ \\
\hline $\begin{array}{l}\text { Particulate size distribution of } \\
\text { feed }\end{array}$ & $\begin{array}{l}\text {-Vendor Info or sieve analysis of material used to prepare } \\
\text { surrogate materials } \\
\text {-Sieve analysis of grab samples collected from drums. }\end{array}$ \\
\hline $\begin{array}{l}\text { Particle size distribution of } \\
\text { conditioned material }\end{array}$ & $\begin{array}{l}\text { Sieve analysis performed grab samples of material to ST-90 } \\
\text { boxes. }\end{array}$ \\
\hline Magnetic metal content of feed & $\begin{array}{l}\text {-Weigh empty drum plus added metal } \\
\text {-Weigh full drum }\end{array}$ \\
\hline $\begin{array}{l}\text { Magnetic metal content of } \\
\text { conditioned material }\end{array}$ & $\begin{array}{l}\text {-Weigh grab sample } \\
\text {-Separate and weigh metal from grab samples of material to ST- } \\
\text { 90 boxes. }\end{array}$ \\
\hline $\begin{array}{l}\text { Homogenization during shredding } \\
\text { and drying of material }\end{array}$ & $\begin{array}{l}\text {-Concentration of tracer material in feed } \\
\text {-Concentration of tracer material in grab samples of material to } \\
\text { ST-90 boxes. }\end{array}$ \\
\hline $\begin{array}{l}\text { Particulate dust emissions from } \\
\text { process vents }\end{array}$ & EPA Method 5 sampling and analysis. \\
\hline Fugitive dust emissions & $\begin{array}{l}\text { Visual inspection and recording in logbook of the presence, } \\
\text { development and implement method onsite to prevent emission. }\end{array}$ \\
\hline Utilities consumption & Electric meter and propane meter readings. \\
\hline Maximum feed rate & $\begin{array}{l}\text { Increase system feed rate until subsystem can no longer operate } \\
\text { correctly. }\end{array}$ \\
\hline $\begin{array}{l}\text { Capacity to process 85 gallon } \\
\text { overpacks }\end{array}$ & $\begin{array}{l}\text { Feed 85-gallon overpacks to system and monitor system for } \\
\text { mechanical failures. }\end{array}$ \\
\hline $\begin{array}{l}\text { Homogenizer Inlet Gas } \\
\text { Temperature }\end{array}$ & Temperature transducer in inlet duct \\
\hline Temperature & Temperature transducer in outlet duct \\
\hline Dust Collector Inlet Pressure & $\begin{array}{l}\text { Pressure transmitter in nuisance dust and homogenizer exhaust } \\
\text { filter inlets }\end{array}$ \\
\hline
\end{tabular}

\subsubsection{Regulatory Data}

In addition to the performance data, facility compliance data regarding the discharge of materials will be collected. These discharge points include air emissions and solids discharge. Since the surrogate wastes are non-hazardous and will be prepared to specific recipe formulations, the processed solids will not be sampled to gather regulatory data. However, the feedstock and pretreated solids will be sampled and analyzed for performance data, as stated above. 
There are two potentially regulated air emissions points, the Nuisance Dust Collection System vent and the Homogenizer Exhaust vent. The level of emissions under the limited demonstration scenario is low enough to constitute a minor source requiring no air permit. No hazardous air pollutants or radionuclides will be discharged and the emissions of criteria pollutants will be well below the minor source limits of 25 tons per year.

\subsubsection{Summary of Test Runs}

The demonstration of the Vortec Materials Pre-Treatment and Conditioning System can be divided into 6 discrete modes of operation, the first being a pretest and common activities and then a series of 5 test runs that are summarized in Table 4.4.4-1. The details of these activities and test runs are given in the following.

Table 4.4.4-1 Test Objectives and Supporting Experiments

\begin{tabular}{|l|l|c|c|c|c|c|}
\hline No. & \multicolumn{1}{|c|}{$\begin{array}{c}\text { Test Objective } \\
\text { Average } \\
\text { Moisture }\end{array}$} & $\begin{array}{c}\text { Test Run 2 } \\
\text { High } \\
\text { Moisture }\end{array}$ & $\begin{array}{c}\text { Test Run 4 } \\
\text { Test Run 3 } \\
\text { High Metals }\end{array}$ & $\begin{array}{c}\text { Test Run 5 } \\
\text { Particulate } \\
\text { Size }\end{array}$ & $\begin{array}{c}\text { 85 Gallon } \\
\text { Overpack } \\
\text { Capacity }\end{array}$ \\
\hline 1 & Drying Performance & $\mathrm{X}$ & $\mathrm{X}$ & $\mathrm{X}$ & $\mathrm{X}$ & \\
\hline 2 & $\begin{array}{l}\text { Tramp Iron Magnet } \\
\text { Performance }\end{array}$ & $\mathrm{X}$ & $\mathrm{X}$ & $\mathrm{X}$ & & \\
\hline 3 & $\begin{array}{l}\text { Particle Size Reduction } \\
\text { Efficiency }\end{array}$ & & & & $\mathrm{X}$ & \\
\hline 4 & $\begin{array}{l}\text { Homogenization } \\
\text { Effectiveness }\end{array}$ & $\mathrm{X}$ & $\mathrm{X}$ & & & \\
\hline 5 & Drum Size Capacity & $\mathrm{X}$ & & $\mathrm{X}$ & $\mathrm{X}$ & $\mathrm{X}$ \\
\hline 6 & $\begin{array}{l}\text { System Maximum } \\
\text { Processing Rate }\end{array}$ & $\mathrm{X}$ & $\mathrm{X}$ & $\mathrm{X}$ & $\mathrm{X}$ \\
\hline 7 & Utilities Consumption & $\mathrm{X}$ & $\mathrm{X}$ & $\mathrm{X}$ & $\mathrm{X}$ & $\mathrm{X}$ \\
\hline 8 & $\begin{array}{l}\text { Efficiency of Dust Emissions } \\
\text { Control System }\end{array}$ & $\mathrm{X}$ & & $\mathrm{X}$ & $\mathrm{X}$ & $\mathrm{X}$ \\
\hline 9 & Fugitive Dust Emission & $\mathrm{X}$ & $\mathrm{X}$ & $\mathrm{X}$ & & \\
\hline 10 & $\begin{array}{l}\text { System Response to Primary } \\
\text { Electrical Power Failure }\end{array}$ & $\mathrm{X}$ & & & & $\mathrm{X}$ \\
\hline
\end{tabular}

\section{Pre-Test and Common Activities}

In this experimental design, it is assumed that each individual piece of equipment will have been checked out during pre-startup activities. Any system deficiencies will have been corrected during this shakedown period. Also, utility connections and the control systems will be tested for functionality prior to initiating the Experiment Sequence.

The Nuisance Dust Collector system (blower, filters) will be operated during each test. The particulate emissions will be directly measured at the Nuisance Dust Collector vent. 


\section{$\underline{\text { Test Run } 1 \text { - Average Moisture Content Feed Testing }}$}

During Test Run 1, the performance of the system will be evaluated with the average moisture content waste, averaging $15 \%$ moisture, the nominal design point of the homogenizer. The feed material for this test will be clean, damp soil in plastic lined drums. The moisture content of the drummed material will be adjusted as closely as possible to $15 \%$ by weight. Every fifth drum will be sampled to determine the average and range of moisture contents in the feed material. Each drum will be placed into the skip hoist by the manipulating arm, fed into the primary shredder, and treated through the remainder of the system. Grab samples of the shredded and dried materials will be collected from the conditioned soil stream prior to the material entering the ST-90 box for disposal. These grab samples will be used to assess the moisture content in the conditioned soil, and the degree of metals separation. The duration of this test is 5 days. The feed rates will be $25 \%, 50 \%, 75 \%$, and $100 \%$ of the maximum processing rate based on mechanical limitations.

The amount of homogenization that occurs during drying will also be evaluated during Test Run 1 . Drums containing a tracer will be fed into the system and grab samples of the conditioned soil will be taken for tracer concentration analysis.

Additionally, during Test Run 1 the ability of the system to meet particulate emission criteria will be evaluated. One hour of flue gas sampling in accordance with EPA Methods 1 through 5 will be performed at the maximum feed rate.

The ability of the back-up electrical power generation subsystem to respond in time to prevent fugitive dust emissions from the system in the event of a primary electrical power failure will also be evaluated during Test Run 1. A some point during the performance of Test Run 1, the main circuit breaker on the electrical power supply to the system will be turned off. The pressures at the inlet to the nuisance dust collector and the homogenizer exhaust dust collector will be measured and recorded at one second intervals during the response period of the back-up generation subsystem. The pressures will be recorded until steady state conditions are achieved in the dust collection subsystems. After steady state conditions are achieved, the main circuit breaker will be turned back on. During the period of time when the main circuit breaker is off, the system will be also visually monitored for fugitive dust emissions.

\section{Test Run 2 - High Moisture Content Feed Testing}

The objective of Test Run 2 is to test the ability of the system to handle a high moisture content waste, $30 \%$ moisture, the maximum design value of the homogenizer. The protocol for Test Run 1 will be repeated. The feed material for this test will be clean, damp soil in plastic lined 55-gallon steel drums. The moisture content of the drummed material will be adjusted as closely as possible to $30 \%$ by weight. Grab samples of every fifth drum will be taken for moisture analysis prior to feeding the materials. Each 
drum will be placed into the skip hoist by the manipulating arm, fed into the primary shredder, and treated through the remainder of the system. Grab samples of the shredded and dried materials will be taken prior to the material entering ST-90 storage box and analyzed for moisture content. In addition, an assessment of the degree of metals separation will be made. The duration of this test is 5 days. The feed rates will be $25 \%, 50 \%, 75 \%$, and $100 \%$ of the maximum processing rate based on mechanical limitations.

The amount of homogenization that occurs during drying will also be evaluated during Test Run 1 . Drums containing a tracer will be fed into the system and grab samples of the conditioned soil will be taken for tracer concentration analysis.

\section{Test Run 3 - High Metals Content Feedstock Testing}

The objective of Test Run 3 is to check the effectiveness of metal removal at the ferrous metal separator. The feed material for this test will be clean, damp soil in plastic lined drums fed to the primary shredder. The drums will have additional scrap metal, rebar and other metallic materials added to the contents. The protocol for Test Runs 1 and 2 will be repeated. Grab samples of every fifth drum will be taken for moisture analysis prior to feeding the materials. Each drum will be placed into the skip hoist by the manipulating arm, fed into the primary shredder, and treated through the remainder of the system. Grab samples of the shredded and dried materials will be collected from the conditioned soil stream prior to the material entering the ST-90 box for disposal. These grab samples will be used to assess the moisture content and metals concentration in the conditioned soil. The duration of this test is 5 days. The feed rates will be $25 \%, 50 \%, 75 \%$, and $100 \%$ of the maximum processing rate based on mechanical limitations.

\section{Test Run 4 - High Particle Size Testing}

The objective of Test Run 4 is to determine the ability of the system to reduce the size of waste particles to a more homogeneous size distribution. The feed material for this test will be clean, damp soil in plastic lined drums fed to the primary shredder. The drums will have additional large particle size materials, such as concrete chunks, added to the contents. At least one drum will be filled with a single cast of concrete. The protocol for Test Runs 1 and 2 will be repeated. Grab samples of every fifth drum will be taken for moisture analysis prior to feeding the materials. Each drum will be placed into the skip hoist by the manipulating arm, fed into the primary shredder, and treated through the remainder of the system. Grab samples of the shredded and dried materials will be collected from the conditioned soil stream prior to the material entering the ST-90 box for disposal. These grab sample will be used to assess the moisture content in the conditioned soil and the degree of particle size reduction. The duration of this test is 5 days. The feed rates will be $25 \%, 50 \%, 75 \%$, and $100 \%$ of the maximum processing rate based on mechanical limitations.

\section{Test Run 5 - 85 Gallon Overpacks}


The objective of Test Run 5 is to confirm the capability of the system to handle 85-gallon overpack containers. During the test, special attention will be given to the operation of the drum manipulator arm, the skip hoist, and the shredders to note any operating irregularities or problems.

The feed to the system during this test will consist of 85-gallon overpack containers filled with clean soil and other debris. The moisture content of the soil in the drums will be set to 30 percent by weight. The system will be brought up to a steady state operating condition and samples collected. Each drum will be placed into the Skip Hoist by the Manipulating Arm, fed into the primary shredder, and treated through the remainder of the system. The duration of this test is 3 days, and the feed rates will be $50 \%$ and $100 \%$ of the maximum processing rate based on mechanical limitations.

\subsubsection{Performance Goals and Test Reports}

The performance goals listed in Table 4.4.5-1 will be validated via the test data plots summarized in Table 4.4.5-2 and test data reports summarized in Table 4.4.5-3.

Table 4.4.5-1 Performance Goals

\begin{tabular}{|l|l|l|}
\hline & & \\
\hline $\begin{array}{l}\text { Homogenizer Drying } \\
\text { Performance }\end{array}$ & $\begin{array}{l}\text { Moisture content of } \\
\text { conditioned material }\end{array}$ & $\begin{array}{l}\leq 5 \% \text { when moisture content } \\
\text { of feed is } \leq 30 \%\end{array}$ \\
\hline $\begin{array}{l}\text { Tramp Iron Magnet } \\
\text { Performance }\end{array}$ & $\begin{array}{l}\text { Ferrous metal content of } \\
\text { conditioned material }\end{array}$ & $\leq 1 \%$ \\
\hline $\begin{array}{l}\text { Waste Size Reduction } \\
\text { Performance }\end{array}$ & Size of conditioned material & $\begin{array}{l}\text { Non-metallic particles } \leq 2 \% \\
\text { Metallic particles } \leq 2 \text { ' x 6" }\end{array}$ \\
\hline Drum Size Capacity & Drum Size, Availability & $\begin{array}{l}\text { Process 55 gal. steel drums } \\
\text { and 85 gal. steel overpacks }\end{array}$ \\
\hline System Processing Rate & Drums processed & $\begin{array}{l}\geq 6 \text { drums per hour at 90\% } \\
\text { availability }\end{array}$ \\
\hline Particulate Removal Efficiency & $\begin{array}{l}\text { Particulate concentration } \\
\text { before and after dust collector }\end{array}$ & $\begin{array}{l}\text { Meet Federal, State, and } \\
\text { Local Standards }\end{array}$ \\
\hline Dust Control Effectiveness & Fugitive dust emissions & None \\
\hline $\begin{array}{l}\text { Back-up Electrical Power } \\
\text { Generation Effectiveness }\end{array}$ & System pressure & $\begin{array}{l}\text { Maintain negative system } \\
\text { pressure for dust control }\end{array}$ \\
\hline
\end{tabular}


Table 4.4.5-2. Test Data Plots

\begin{tabular}{|c|c|c|c|}
\hline $\begin{array}{c}\text { Plot (dependent vs. } \\
\text { independent variable) }\end{array}$ & $\begin{array}{l}\text { Test Objectives } \\
\text { Supported }\end{array}$ & Data Series on Plot & Comparison/Conclusions \\
\hline $\begin{array}{l}\text { Conditioned material } \\
\text { moisture content versus } \\
\text { feed rate for various feed } \\
\text { conditions }\end{array}$ & $\begin{array}{l}\text { 1. Homogenizer } \\
\text { Drying } \\
\text { Performance }\end{array}$ & $\begin{array}{l}\text { 1. High moisture feed } \\
\text { 2. Average moisture feed } \\
\text { 3. High metals feed } \\
\text { 4. Large particles in feed }\end{array}$ & $\begin{array}{l}\text { 1. Effects of material characteristics } \\
\text { on the moisture content in the } \\
\text { conditioned soil. } \\
\text { 2. Effects of feed rate on the } \\
\text { moisture content in the conditioned } \\
\text { soil. }\end{array}$ \\
\hline $\begin{array}{l}\text { Water removal efficiency } \\
\text { versus feed rate }\end{array}$ & $\begin{array}{l}\text { 1. Homogenizer } \\
\text { Drying } \\
\text { Performance }\end{array}$ & $\begin{array}{l}\text { 1. High moisture feed } \\
\text { 2. Average moisture feed } \\
\text { 3. High metals feed } \\
\text { 4. Large particles in feed }\end{array}$ & $\begin{array}{l}\text { 1. Effects of material characteristics } \\
\text { on the moisture removal efficiency of } \\
\text { homogenizer. } \\
\text { 2. Effects of feed rate on moisture } \\
\text { removal efficiency of homogenizer. }\end{array}$ \\
\hline $\begin{array}{l}\text { Metal Content of } \\
\text { conditioned material vs. } \\
\text { feed rate of material and } \\
\text { metal removal efficiency }\end{array}$ & $\begin{array}{l}\text { Tramp Iron } \\
\text { Magnet } \\
\text { Performance }\end{array}$ & $\begin{array}{l}\text { 1. High moisture feed } \\
\text { 2. Average moisture feed } \\
\text { 3. High metals feed }\end{array}$ & $\begin{array}{l}\text { 1. Effects of feed moisture content } \\
\text { on metals content in conditioned } \\
\text { material. } \\
\text { 2. Effects of feed rate on metals } \\
\text { content of conditioned material. } \\
\text { 3. Impact of large metal pieces in } \\
\text { feed on metals content of the } \\
\text { conditioned material. }\end{array}$ \\
\hline $\begin{array}{l}\text { \% passing a sieve size of } \\
\text { vs. particle/sieve size }\end{array}$ & $\begin{array}{l}\text { 2. Particle Size } \\
\text { Reduction } \\
\text { Efficiency }\end{array}$ & $\begin{array}{l}\text { 1. Feed material } \\
\text { 2. } 25 \% \text { maximum feed rate } \\
\text { 3. } 50 \% \text { maximum feed rate } \\
\text { 4. } 75 \% \text { maximum feed rate } \\
\text { 5. } 100 \% \text { maximum feed } \\
\text { rate }\end{array}$ & $\begin{array}{l}\text { Effect of feed rate on particle size on } \\
\text { large pieces passing through system. }\end{array}$ \\
\hline $\begin{array}{l}50 \% \text { less than particle } \\
\text { size vs. feed rate. }\end{array}$ & $\begin{array}{l}\text { 2. Particle Size } \\
\text { Reduction } \\
\text { Efficiency }\end{array}$ & 1. Data from Experiment 4 & $\begin{array}{l}\text { Determine the impact of feed rate on } \\
\text { particle size reduction efficiency. }\end{array}$ \\
\hline $\begin{array}{l}\text { Tracer concentration in } \\
\text { conditioned material vs. } \\
\text { sampling time }\end{array}$ & $\begin{array}{l}\text { 3. Homogenization } \\
\text { Effectiveness }\end{array}$ & $\begin{array}{l}\text { Two plots, high and average } \\
\text { moisture feeds each } \\
\text { containing: } \\
\text { 1. } 25 \% \text { maximum feed rate } \\
\text { 2. } 50 \% \text { maximum feed rate } \\
\text { 3. } 75 \% \text { maximum feed rate } \\
\text { 4. } 100 \% \text { maximum feed } \\
\text { rate }\end{array}$ & Illustrate blending effect of system. \\
\hline
\end{tabular}




\begin{tabular}{|l|l|l|l|}
\hline $\begin{array}{l}\text { Homogenization } \\
\text { Effectiveness vs. feed }\end{array}$ & 3. Homogenization & $\begin{array}{l}\text { 1. High moisture feed } \\
\text { 2. Average moisture feed }\end{array}$ & $\begin{array}{l}\text { Effect of feed rate on } \\
\text { Homogenization Effectiveness of } \\
\text { system. }\end{array}$ \\
\hline $\begin{array}{l}\text { Dust collector inlet } \\
\text { pressure vs. time }\end{array}$ & $\begin{array}{l}\text { 10. System } \\
\text { Response to } \\
\text { Primary Electrical } \\
\text { Power Failure }\end{array}$ & $\begin{array}{l}\text { 1. Dust Collector inlet } \\
\text { pressure }\end{array}$ & $\begin{array}{l}\text { Determine maximum pressure during } \\
\text { back-up power generation subsystem } \\
\text { response time. }\end{array}$ \\
\hline
\end{tabular}

Table 4.4.5-3 Test Data Reports

\begin{tabular}{|l|l|l|l|}
\hline \multicolumn{1}{|c|}{$\begin{array}{c}\text { Data Reported in } \\
\text { Table }\end{array}$} & \multicolumn{1}{|c|}{$\begin{array}{c}\text { Test Objectives } \\
\text { Supported }\end{array}$} & Other details provide & \multicolumn{1}{c|}{ Conclusions } \\
\hline $\begin{array}{l}\text { Number of drums } \\
\text { processed at each drum } \\
\text { feed, number of failure } \\
\text { events for Experiments }\end{array}$ & 5. Drum Size Capacity & $\begin{array}{l}\text { Description of failure } \\
\text { events. }\end{array}$ & $\begin{array}{l}\text { A sufficient number of } \\
\text { drums were processed to } \\
\text { demonstrate a 90\% } \\
\text { system availability for both } \\
\text { drum sizes. }\end{array}$ \\
\hline $\begin{array}{l}\text { Number of drums } \\
\text { processed at each drum } \\
\text { feed, number of failure } \\
\text { events for Experiments }\end{array}$ & Processing Rate & $\begin{array}{l}\text { Description of failure } \\
\text { events. }\end{array}$ & $\begin{array}{l}\text { A sufficient number of } \\
\text { drums were processed to } \\
\text { demonstrate a 90\% } \\
\text { system availability for } \\
\text { each feed rate. }\end{array}$ \\
\hline $\begin{array}{l}\text { Summary of total utilities } \\
\text { consumed, amount of } \\
\text { material processed, } \\
\text { hours of operation. }\end{array}$ & 7. Utilities Consumption & $\begin{array}{l}\text { Calculation of the total } \\
\text { amount of material } \\
\text { processed. }\end{array}$ & $\begin{array}{l}\text { The resulting utilities } \\
\text { consumption rate per unit } \\
\text { of material processed. }\end{array}$ \\
\hline $\begin{array}{l}\text { Location, time, estimate } \\
\text { emission rate, and } \\
\text { corrective action. }\end{array}$ & 9.Fugitive Dust Emission & $\begin{array}{l}\text { Details of the corrective } \\
\text { action }\end{array}$ & $\begin{array}{l}\text { List of potential fugitive } \\
\text { dust emission points }\end{array}$ \\
\hline
\end{tabular}




\subsection{Plant Location}

The Vortec demonstration facility will be located on the western edge of the PGDP on the Paducah Site. The Paducah Site is in McCracken County, Kentucky, approximately $24 \mathrm{~km}$ (15 mi) west of the city of Paducah and about $5.6 \mathrm{~km}(3.5 \mathrm{mi})$ south of the Ohio River. The Paducah Site is a DOE reservation consisting of a 582-ha (1,437-acre) DOE-managed site and an 804-ha (1,986-acre) wildlife management area leased to the Commonwealth of Kentucky. The Paducah Site has included an active uranium enrichment facility since 1951. PGDP occupies a 303-ha (748-acre) complex at the center of the Paducah Site and is surrounded by a security fence. United States Enrichment Corporation (USEC), a private corporation, operates the uranium enrichment facilities at PGDP. Figure 4.5-1 depicts the location of the demonstration test facility relative to the gaseous diffusion plant.

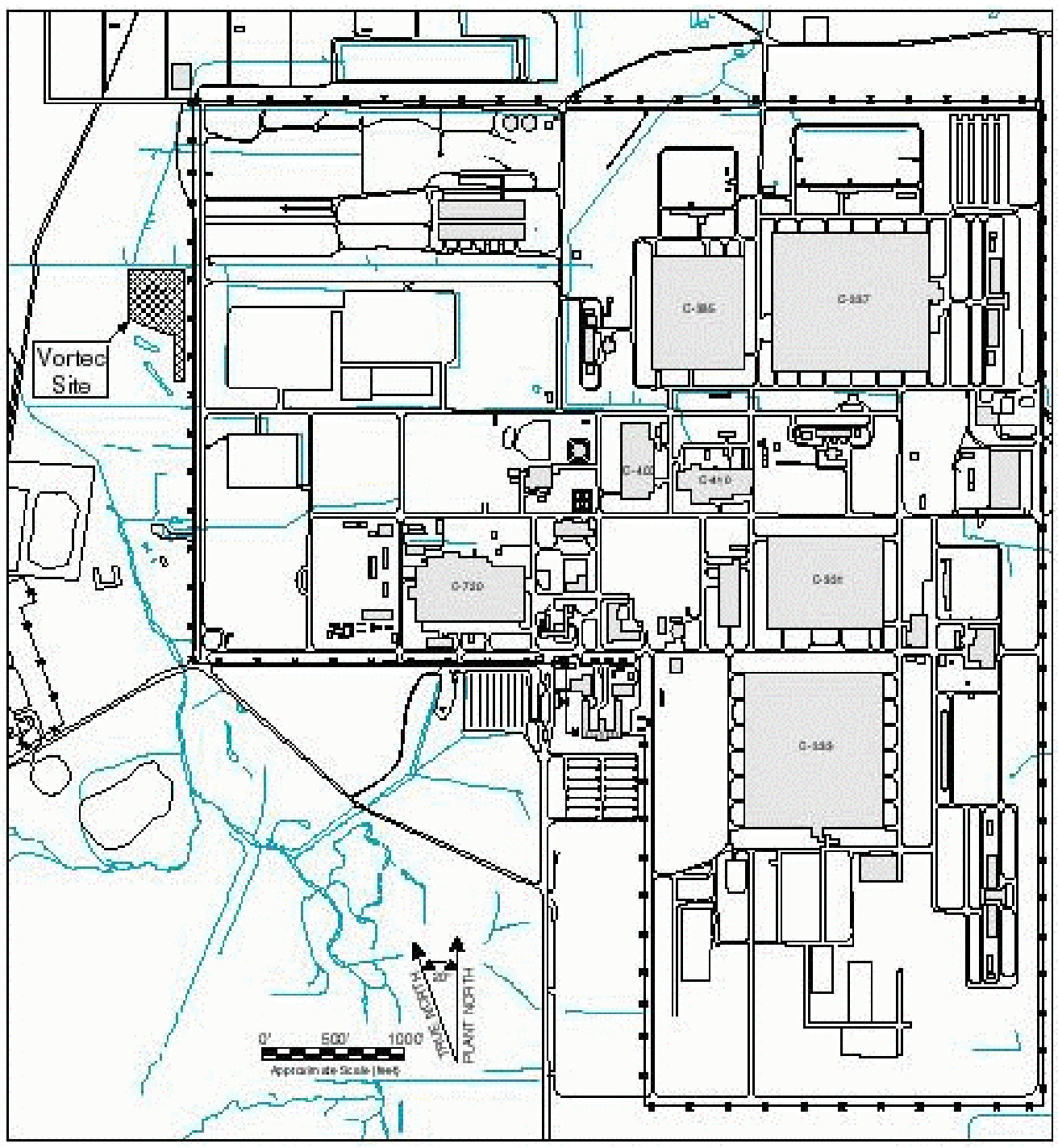

Figure 4.5-1 Location of Demonstration Facility Relative to Gaseous Diffusion Plant 


\subsection{Regulatory AND PERMitTing Status}

\subsubsection{Vitrification Plant- RD\&D and Air Permits}

DOE, with Vortec's assistance, had prepared a draft application for a Research Development and Demonstration (RD\&D) permit in order to demonstrate the vitrification plant. This permit was required prior to construction of the vitrification plant and would cover operations of the demonstration. The RD\&D permit was delayed pending finalization of the EA. As of early 2000, the RD\&D permit was in the final stages of revision to conform to agency comments on a draft application. The final, formal application package to be submitted was to consist of three sections, Permit Application, Demonstration Test Plan, and Risk Assessment Protocol. As of early 2000, the application document was complete, DOE had completed the Risk Assessment Protocol and the EPA SITE Program was preparing the Demonstration Test Plan. EPA withdrew their support for the Demonstration Test Plan when DOE decided to discontinue the vitrification portion of the project.

An air permit for the demonstration of the vitrification plant had been issued in 1996. This permit was required before the site preparation work performed in 1997-1998 could proceed.

\subsubsection{Limited Demonstration Plant Permit Requirements}

The permitting situation has changed with the change of scope from demonstration of the innovative vitrification plant to the limited demonstration. An RD\&D permit is not required for the current effort since no hazardous materials will be processed. However, DOE-Paducah had planned to continue with the RD\&D permitting effort in order to preserve the future ability to process RCRA waste through the Soil Preparation and Conditioning facilities. Following the request of the Kentucky Department of Waste Management to either finalize the application or withdraw it, DOE-Paducah has decided to withdraw the RD\&D permit application.

Generally, the State may require a separate air permit prior to construction, regardless of the hazardous nature of materials processed. However, preliminary calculations by DOE-Paducah indicate that an air permit is not required for construction and demonstration of the soil preparation and feed handling facilities. The basis for this conclusion is that no hazardous air pollutants or radionuclides would be discharged and the emissions of criteria pollutants would be well below the minor source limits. The Kentucky Department for Environmental Protection Division of Air Quality issued an air permit for the Phase 3 Demonstration in May 1996. Among the permit conditions was a requirement that the air permit would expire if construction was commenced and then stopped for 18 months. Based on this permit condition, upon the request of the Division for Air Quality, DOE-Paducah has decided to withdraw the existing air permit.

Both a RCRA program permit (analogous to the RD\&D permit) and an air permit will be required before the Soil Preparations and Conditioning Facilities can be used to process low level waste, mixed waste or hazardous waste. 


\subsection{CONCLUSIONS}

\subsection{CONCLUSIONS FROM ENVIRONMENTAL ASSESSMENT}

The Environmental Assessment concluded that demonstrating the Vortec pretreatment/vitrification process would cause no significant impact to the surrounding community or the environment. The impacts of constructing and operating the Vortec CMS®demonstration facility at the Paducah Site are summarized as follows.

- $\quad$ Emissions of criteria air pollutants would not exceed standards or incremental levels. Emissions of hazardous air pollutants (HAPs) would be at a level less than that requiring classification of the facility as a major source of HAPs. Emission levels would be very low and not pose risks of adverse effects to workers or the public.

- $\quad$ Noise levels experienced by the nearest resident would not be distinguishable from backgroundlevel noise.

- $\quad$ Release of occasional small quantities of wastewater to the PGDP outfall would have negligible impacts on surface water, even under accident conditions.

- $\quad$ Off-site recreational activity at the nearby West Kentucky Wildlife Management Area would not be disturbed by construction or operation of the Vortec facility.

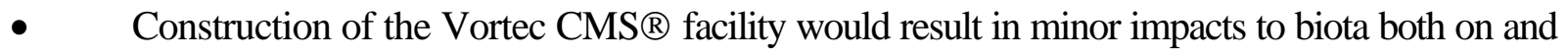
off the site. Very few polychlorinated biphenyls (PCBs) would be released during normal operations or accidents; levels would be too low to affect human water supplies or to measurably increase the bioaccumulation already present in Ohio River from upstream sources.

- $\quad$ Radiological doses to involved workers, noninvolved workers, and members of the public from Vortec facility operations and from accidents would be very low and well below applicable limits and guidelines.

- If a fire and propane tank explosion were to occur, heat and overpressure effects might kill or injure a worker but would not harm the nearest member of the public.

- $\quad$ A highly unlikely, but possible, accidental release of waste drum contents might have a lethal effect on a worker but would not be life-threatening to the public.

- $\quad$ Standard accident rates for construction and manufacturing indicate that the chance of a worker fatality during construction and operation is less than 1 in 100. In a workforce of up to 50 individuals, the number of injuries involving lost workdays is expected to be 1 to 2 . 
- $\quad$ The landfill at the Paducah Site has adequate storage capacity, should the Vortec CMS® product meet applicable waste acceptance criteria.

- $\quad$ The construction and operation of the Vortec CMS ${ }^{\circledR}$ would not result in any adverse impact to minority or low-income communities.

- The cumulative impacts of criteria air emissions from the Vortec facility, nearby facilities, and the ambient background would not exceed standards or increment levels. PCBs, if handled at the Vortec facility, would result in a maximum ground level concentration that would be 700 times less than the concentration believed to cause a cancer risk of one in 1 million. When potential radiological doses from Vortec CMS ${ }^{\circledR}$ emissions are added to other regional emissions, the calculated probability of a cancer fatality for the region exposed to all sources would be one in 1 million.

\subsection{IMPLEMENTATION BARRIERS}

The primary barriers to implementing new technology for DOE waste management applications are generally technical, cost, regulatory or political in nature.

Technical barriers can generally be overcome by good engineering and design practices. The capital and operating costs of the technology, however, must be traded off against the system performance requirements to meet environmental statutes and regulations. Political factors, such as waste clean-up priorities and the method by which waste clean-up contracts are administered will also impact the overall probability of successful technology implementation.

This project represents a good example where the regulatory and political factors have played a dominant role in delaying the implementation of a sound, cost-effective technology. In this regard, Table 5.2-1 is a summary of the history of the project starting from the time it was decided to proceed with the Limited Demonstration at the PGDP.

In reviewing the history events, it is evident that project delays were caused by compliance with regulatory requirements precipitated by political factors. The political factors, in this case, were caused by intervention of a single environmental activists into the project. The vehicle by which this occurred was through membership on the Specific Advisory Board (SSAB). The procedure by which the project was interrupted was the filing of a law suit on the basis that DOE was not following its own NEPA procedures.

The Vortec vitrification process has been classified by the U.S. EPA as a Subcategory X treatment process. Opposition to the vitrification technology by the environmental activists was based on the fact that it is a thermal technology, and therefore, meant it was "incineration." Identification of a thermal technology as incineration is problematic in this country because of the irrational hysteria that has developed over the past several years. 


\section{Table 2-1. Vortec Vitrification Project History}

- January 1996 - Contract modified to include scope of Phase III Demonstration Project.

- February 1997- Citizen files complaint in federal court against DOE under National Environmental Protection Act. DOE agrees to perform an Environmental Assessment for the RD\&D project.

- February 1997- KDWM issues RD\&D permit.

- March 1997- KDWM rescinds its issuance of RD\&D permit which returns permit to draft status.

- June 1997- DOE and citizen agree to a settlement. DOE agrees to prepare an Environmental Assessment for the project pursuant to DOE's NEPA polic y and to stop certain work on the project.

- 1998 - Vortec completes site work

- 1997 - 1998 - Equipment valued at approximately \$6 million is purchased and delivered to the site.

- October 18, 1999- KDWM provides a second preliminary draft permit for comment

- December 1999- EA preparation complete. The EA recommended a finding of no significant impact (FONSI) to the environment for the Vortec Demonstration project.

- February 16, 2000- Draft revision to RD\&D RCRA Permit Application issued for internal review prior to submittal to KDWM.

- March 2000 - FONSI approved and issued by DOE.

- March 2000 - Citizen again files suit, alleging the EA and FONSI are flawed and requesting a sitewide Environmental Impact Statement (EIS).

- April 2000 - DOE made decision to perform a limited demonstration to make use of its investment in equipment.

- May 2000 - DOE issues a draft change of scope to the Limited Demonstration and instructs Vortec to prepare a proposal for a limited demonstration.

- June 2000 - Limited Demonstration cost and technical proposals submitted.

- August 2000 - Contract is modified to include funds to complete a Limited Demonstration. Scope of work is not definitized pending settlement of the citizen's suit.

- November 2000 - Second citizen lawsuit is settled allowing Limited Demonstration to proceed.

- May 2001 - DOE commits to completing engineering for Limited Demonstration Project.

Even though thermal destruction is perhaps the safest and most effective means of treating organic containing wastes, the Vortec vitrification technology as well as other promising thermal treatment processes are not being used by DOE because DOE has been unsuccessful in defending the use of these technologies against environmental activists who challenge DOE's environmental procedures.

As a result of these political and regulatory failures, DOE has now implemented technology research into "non-thermal" treatment processes that are likely to be not as effective or safe as thermal treatment's processes. Re-direction of Vortec demonstration program to just the front end of the process (i.e., the Material Handling and Conditioning System) is a reflection of the implementation challenges that have faced DOE with thermal technologies. 


\subsection{IMPLEMENTATION OF THEMATERIAL HANDLING AND CONDITIONING SYSTEM}

Vortec has decided to focus its technology demonstration efforts on the Paducah Gaseous Diffusion Plant. Successful operational implementation of the Material Handling and Conditioning System is likely to occur for the following reasons:

Vortec has decided to focus on implementation of the limited demonstration at Paduca. The primary reasons for making this selection included:

8. DOE/Paducah's previous support for the integrated pretreatment/vitrification demonstration.

9. DOE/Paducah's commitment to continue support for the Limited Demonstration.

10. A commitment from the United States Enrichment Corporation (USEC) for cost share participation contingent upon development of a favorable path forward for securing the necessary permits and a waste management contract which will utilize the waste handling and conditioning system.

11. A favorable response from Bechtel Jacobs Corporation to pursue a project which would target the processing of drummed legacy waste on a near-term basis.

12. The existing infrastructure at Paduah (i.e., site preparation) to accommodate the limited demonstration.

13. Expected favorable support from the Kentucky regulators for the project.

14. Resolution of the law suit between the principals of the SSAB and DOE to allow the Limited Demonstration to proceed.

The bottom line conclusion from cost benefit analyses performed by Vortec and others is that the Material Handling and Conditioning System will provide a safer, quicker and cheaper method of preparing and packaging waste for final treatment (if required) and disposal.

With adequate funding provided by DOE, the proposed demonstration project can be completed in FY 2002.

\subsection{RECOMMENDATIONS}

The following recommendations are provided with regard to demonstration of the Material Handling and Conditioning System:

1. The Paducah Gaseous Diffusion Plant should be used as the test facility for the Limited Demonstration Test Program.

2. Adequate funding should be provided to allow completion of the demonstration in FY 2002. 


\subsection{REFERENCES}

1. Hnat, J.G., Ph.D, Patten, John, Ph.D, Jian, Christopher "Vitrification of Waste Streams Containing RCRA Metal Compounds" prepared for Environmental Protection Agency's Workshop “Arsenic and Mercury: Removal, Recovery, Treatment and Disposal, August 1992.

2. Shearer, Teri, etal "Vitrification of Heavy Metal Contaminated Soils with Vortec Corporation's Combustion \& Melting System (CMS®)" I \& EC Special Symposium American Chemical Society, Atlanta, GA September 21-23, 1992.

3. Hnat, J.G., Ph.D, Patten, John, Ph.D, "Vitrification and Soils Stabilization”, presented at: Environmental Remediation in New Jersey, Newark November 9, 1994.

4. Hnat, J.G., Ph.D, Patten, John, Ph.D, Jian, Christopher, “Application of the Vortec Combustion System for Demilitarization/Disposal of Energetic/Chemical Materials, Demilitarization Symposium, Stouffer Concourse Hotel, Arlington, Virginia. May 23-25, 1994.

5. Jetta, Norman W., Patten, John, Ph.D, Hnat, J.G., Ph.D, "Innovative Vitrification for Soil Remediation”, METC -Environment Technology Through Industry Partnership, October 3-5, 1995.

6. Hnat, J.G., Ph.D, Patten, John, Ph.D, Jetta, Norman W., "Innovative Vitrification for Soil Remediation", METC-Industry Partnerships to Deploy Environmental Technology, October 22-24, 1996.

7. Jetta, Norman W., Patten, John, Ph.D, Hnat, J.G., Ph.D, "Innovative Vitrification for Soil Remediation”, Prepared for WM '96 Tucson, AZ, February 25-29, 1996.

8. Hnat, J.G., Ph.D, Patten, John, Ph.D, Jetta, Norman W., "Innovative Vitrification for Soil Remediation”, Waste Management Conference (1998), March 1-5, 1998.

9. Hnat, J.G., Ph.D, Patten, John, Ph.D, Jetta, Norman W., "High Throughput Vitrification for DOE Waste Remediation",

10. Hnat, J.G., Ph.D, Pineda, Marilyn, Detwiler, Donald, "Innovative Pretreatment and Vitrification Technologies for Waste Remediation”, Abstract Industry Partnerships for Environmental Science and Technology Conference, National Energy Technology Laboratory, Morgantown, West Virginia, October 17-19, 2000 


\subsection{BIBLIOGRAPHY}

1. Vortec Corporation, "Phase 1 Final Report Innovative Fossil Fuel Fired Vitrification Technology for Soil Remediation", for U. S. Department of Energy, January 31, 1994

2. Vortec Corporation, "Phase 2 Summary Report Innovative Fossil Fuel Fired Vitrification Technology for Soil Remediation”, for U. S. Department of Energy, October 6, 1995

3. Hnat, R. K., "Limited Demonstration Operational Plan for the Vortec-Paducah Material Handling System", Topical Report, July 2001.

4. Simpson, J. C., 'Limited Demonstration Test Plan for the Vortec-Paducah Material Handling System", Topical Report, July 2001. 


\subsection{LIST OF ACRONYMS AND ABBREVIATIONS}

ACI

APC

ASCE

BJC

BTU

CFR

$\mathrm{CMS}{ }^{\circledR}$

CRV

CY

DOD

DOE

DRE

DTP

EA

EPA

FONSI

FUSRAP

FY

HASP

HEPA

INEL

IUOE

LLW

MCC

$\mathrm{MH} / \mathrm{C}$

MLLW

MMBTU

NEMA

NEPA

NETL

O\&M

ORP

PCB

PCT

PGDP

PHA
American Concrete Institute

Air Pollution Control

American Society of Civil Engineers

Bechtel Jacobs Corporation

British Thermal Unit

Code of Federal Regulation

Cyclone Melting System

Counter-Rotating Vortex

Calendar Year

Department of Defense

Department of Energy

Destruction \& Removal Efficiency

Demonstration Test Plan

Environmental Assessment

Environmental Protection Agency

Finding of No Significant Impact

Formally Utilized Sites Remedial Action Program

Fiscal Year

Health and Safety Plan

High Efficiency Particulate Assembly

Idaho National Engineering Laboratory

International Union of Operating Engineers

Low Level Waste

Motor Control Center

Material Handling \& Conditioning

Mixed Low Level Waste

Million BTU

National Electrical Manufacturer Association

National Environmental Policy Act

National Energy Technology Laboratory

Operating \& Maintenance

Oxidation-Reduction Potential

Polychlorinated Biphenols

Product Consistency Test

Paducah Gaseous Diffusion Plant

Process Hazards Analysis 
PLC

QAPP

RCRA

RD\&D

SDC

SMS

STAAD

STC

TCE

TCLP

TEFC

TRU

TSCA

UBC

UCRL

U-PARC

USEC

WESP
Process Logic Control

Quality Assuredness Preparedness Plan

Resource Conservation and Recovery Act

Research, Development \& Demonstration

Structural Design Criteria

Paducah DOE Support Contractor

Computer Program Applied by STAAD Corporation

Society for Technical Communication

Trichloroethylene

Toxicity Characteristic Leaching Procedure

Totally Enclosed Fan Cooled

Transuranic

Toxic Substance Control Act

Uniform Building Code

University of California Research Laboratory

University of Pittsburgh Applied Research Center

United States Enrichment Corporation

Wet Electrostatic Precipitator 
Vortec Corporation NETL Phase 3 Final Report July 12, 2001

Page No. 67

\subsection{APPENDICES}

No appendices are included with this report. 